Orthopäde 2022 $51: 307-324$

https://doi.org/10.1007/s00132-022-04235-8

Angenommen: 14. Februar 2022

Online publiziert: 3. März 2022

(c) Der/die Autor(en) 2022

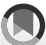

Check for
updates

\section{Therapeutische Injektion und Manuelle Medizin beim tiefen Rückenschmerz}

\author{
Bimodale Synergien zwischen Evidenz und Empirie
}

\author{
Uwe H. W. Schütz', \\ 'Schmerztherapie und Rheumatologie, Orthopädie am Grünen Turm, Ravensburg, Deutschland \\ ${ }^{2}$ Klinik für Diagnostische und Interventionelle Radiologie, Universitätsklinikum Ulm, Ulm, Deutschland
}

Zusammenfassung

\section{In diesem Beitrag}

- Manuelle Medizin bei TRS: Definition, Indikation, Evidenz

Evidenz der MM • Kasuistik-Teil 2:

3-Ebenen-Diagnose und Erstbehandlung

- Therapeutische Lokalinterventionen beim TRS: Techniken, Effektivität, Evidenz Neuraltherapeutische Infiltrationen und Injektionen beim TRS • Epidurale Injektionen (EPI) beim TRS - Kasuistik-Teil 3: Re-Assessment nach 10 Tagen • Facettengelenkinterventionen ( $\mathrm{FGI}$ ) beim TRS • Interventionen am Sakroiliakalgelenk beim TRS - Sonstige interventionelle Verfahren beim TRS • Kasuistik-Teil 4: Re-Assessment, 4. Woche

- Zusammenfassung

- Schlussfolgerungen

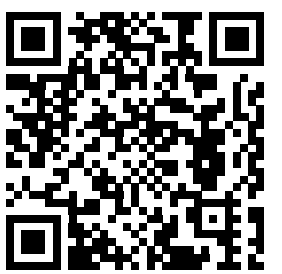

QR-Code scannen \& Beitrag online lesen

\begin{abstract}
Hintergrund: Angelehnt an die zielorientierte Therapieplanung und -führung in der Versorgung chronisch Rheumakranker, plädiert der Autor bei Patienten mit (chronischem) degenerativem tiefem Rückenschmerz (TRS), orientiert an den differenzialtherapeutischen Grundsätzen der Manuellen Medizin (MM), und unter Kenntnis von Techniken und Evidenzen therapeutischer Lokalinfiltrationen (TLI), für die Etablierung eines strukturierten mechanismenbasierten Therapiekonzeptes im Sinne des "treat to target" (T2T) im ambulanten schmerztherapeutischen Versorgungsbereich.

Diagnostik: Dies setzt eine konsequente (Primär-)Diagnostik mit Schmerzanalyse unter der Prämisse, dass TRS, wenn strukturell-funktionell bedingt, immer spezifisch ist, voraus. Eine breite biopsychosoziale Anamnese und strukturbezogene klinischbildgebende (Ausschluss-)Diagnostik mit funktioneller Differenzierung nach MMGrundsätzen und ggf. interventionelle Blocks, sollten am Ende zur Formulierung einer 3-Ebenen-Diagnose als Voraussetzung zu einer mechanismenbasiert-zielorientierthierarchischen Stufentherapie bei TRS führen. Diese wird in diesem Artikel pragmatisch fallorientiert, unter Implementierung von Techniken und Evidenzen der TLI und MM, vorgestellt.
\end{abstract}

\section{Schlüsselwörter}

Chronische Schmerzen · Injektion · Lumbago · Sakroiliakalgelenk · Wirbelsäule
Die Kenntnis der Mechanismen der Schmerzphysiologie sowie die Beherrschung der Methoden der Manuellen Medizin und der therapeutischen Lokalinterventionen, ermöglicht dem ärztlichen Therapeuten, nach suffizienter Diagnostik der primären Schmerzgeneratoren, einen mechanismenbasierten, stadiengerechten synergistisch sinnvollen bi-/multimodalen Methodenter und chronischer, spezifischer tiefer Rückenschmerzen. Am Ende der Behandlung kann eine relevante anhaltende Schmerzreduktion als Voraussetzung für die Rekonditionierung der neuromotorischen Systeme stehen. einsatz zur gezielten Behandlung aku-

\section{Einleitung}

Neben der medikamentösen Systemtherapie sind die Techniken zur Durchführung therapeutischer Lokalinfiltrationen (TLI) und die Methoden der Manuellen Medizin (MM) die wichtigsten Werkzeuge zur Behandlung des tiefen Rückenschmerzes (TRS) in der ambulanten Versorgung. Sie werden von Ärzten verschiedener Fachrichtungen angewandt (z.B. Orthopäden und Unfallchirurgen, Spezielle Schmerztherapeuten, Neurologen, Anästhesisten, Allgemeinmediziner, Rheumatologen).

In der Schulmedizin ist die Stellung der korrekten Diagnose Voraussetzung für eine suffiziente Behandlung des TRS. Hierbei 
geht es in der Praxis jedoch nicht um die simple Auswahl einer annähernd passenden Diagnose aus dem ICD-Katalog, sondern um die Formulierung einer Funktionsdiagnose, welche die individuell vorliegende Ursache der TRS des Patienten wieder-

\section{Abkürzungen}

ACLS "Advanced cardiac life support"

ASIPP "American Society of Interventional

Pain Physicians"

$B \ddot{K} \quad$ Bundesärztekammer

$B M I \quad$ Body-Mass-Index

Btx Botulinumtoxin

BV Bildverstärker (Bildwandler)

CGRP "Calcitonin-gene related peptide"

$D A A O$ Deutsch-Amerikanische Akademie für Osteopathie e.V.

DGAI Deutsche Gesellschaft für Anästhesiologie und Intensivmedizin e.V.

DGMM Deutsche Gesellschaft für Manuelle Medizin e. V.

EPI Epidurale Injektion

FG Facettengelenk

FGI Interventionen am Facettengelenk

FGS Facetten(gelenk)syndrom

GK Glukokortikoide

HVLA "High velocitiy low amplitude"

IDT Intradiskale Interventionen

IGOST Interdisziplinäre Gesellschaft für orthopädische/unfallchirurgische und allgemeine Schmerztherapie e.V.

IL Interleukin

INR International Normalized Ratio

IVR Intervertebralraum

KHK Koronare Herzkrankheit

LA Lokalanästhetikum

LSIR Lumbosakroiliakalregion

LSPA Lumbale Spinalnervanalgesie

LWS Lendenwirbelsäule

MIP Mobilität, Irritation, Provokation

MM Manuelle Medizin

MPSS Mainzer Stadienmodell der Schmerz-

Chronifizierung

PRP "Platteled enriched plasma"

PRT Periradikuläre Therapie

RFN Radiofrequenz-Neurotomie

ROM "Range of motion"

SAMM Schweizerische Ärztegesellschaft für

Manuelle Medizin

SIG Sakroiliakalgelenk

SIPS Spina iliaca posterior superior

SNR "Signal-noise-ratio"

T2T "Treat to target"

TLA Therapeutische Lokalanästhesie

TLI Therapeutische Lokalinfiltration/-

injektion

Tumornekrosefaktor

Tiefer Rückenschmerz

Visuelle Analogskala

„Wide dynamic range neuron"

Zentrales Nervensystem gibt. Damit dies dem Behandler überhaupt gelingen kann, muss dieser die Mechanismen der Schmerzentstehung und -verarbeitung auf Basis eines vertieften Kenntnisstandes hinsichtlich funktioneller Neuroanatomie und -physiologie kennen und verstanden haben.

Anhand eines klinischen Fallbeispiels soll in diesem Artikel dem Leser das auch für den ausgebildeten Facharzt durchaus sehr anspruchsvolle Ziel der Überführung der sehr theorielastigen, neurophysiologischen Erkenntnisse zum TRS (seien sie nun akut oder chronisch, unspezifisch [11] oder spezifisch [34]) in eine alltagstaugliche ursachenbasierte Funktionsdiagnose aufgezeigt werden, auf der erst dann die Etablierung und zielorientierte stadiengerechte mechanismenbasierte (Schmerz-)Therapie bzw. Auswahl und Kombination von Verfahrensweisen der TLI und MM möglich wird.

\section{Tiefer Rückenschmerz}

\section{Neuroanatomie}

Bei der neuroanatomischen Betrachtung der Lumbosakroiliakalregion (LSIR) fällt eine hohe Innervations- und Nozizeptordichte der (peri-)vertebralen Strukturen auf [40]. Die verschiedenen Gewebe weisen, mit Ausnahme des Nucleus pulposus, allesamt nozizeptive Fasern auf, welche unter adäquater Reizung (thermisch, mechanisch etc.) Erregungen des nozizeptiven Systems generieren (- Tab. 1). Ist der Nucleus pulposus als größtes avaskuläres humanes Gewebe primär kein Ort möglicher Nozizeption, so ändert sich dies aufgrund einsetzender Neovaskularisation, sobald es zu einer Bandscheibenschädigung gekommen ist [21], was die hohe Rezidivrate bzw. Vulnerabilität bezüglich des TRS bei vorliegenden Diskopathien mit erklären mag.

\section{Neurophysiologische Mechanismen der Schmerzentstehung und -verarbeitung}

In den letzten beiden Dekaden haben die konsequenten translationalen Bemühungen von manualmedizinischen (Schweizerische Ärzte- bzw. Deutsche Gesellschaft für Manuelle Medizin SAMM/DGMM) und schmerztherapeutischen Ärztegesellschaften (IGOST: Inter- disziplinäre Gesellschaft für orthopädische/unfallchirurgische und allgemeine Schmerztherapie) zur Zusammenführung der Erkenntnisse der neurophysiologischen Grundlagen und der klinischen Schmerzforschung zu einem vertieften Verständnis der Mechanismen der Schmerzentstehung und -verarbeitung geführt, auf welche sich auch u.a. die moderne MM stützt. Zum Verständnis des vorliegenden Artikels werden die diesbezüglich etablierten Fachbegriffe und zugrundeliegenden Mechanismen vorausgesetzt, welche in der einschlägigen Fachliteratur zum chronischen TRS [52, $66,75,81]$ und auch im Beitrag H. Schnell et al. dieses Themenheftes („Die segmentale und somatische Dysfunktion. Wie funktioniert Manuelle Medizin?") als Erklärungsmodell der Wirkungsweise der MM auf neurophysiologischer Ebene, aufgezeigt werden. Diese ineinandergreifenden Mechanismen umfassen von der einfachen akuten Nozizeptoraktivierung bis hin zur komplex-chronischen globalen Hyperalgesie u.a.:

- (nozireaktive) motorische Systemaktivierung (Nozireaktion/-reflex)

- periphere Sensibilisierung, neurogene Inflammation, primäre Hyperalgesie

- afferente Konvergenz via multirezeptivem Hinterhornneuron (WDRNeuron „wide dynamic range neuron" = spinothalamisches Konvergenzneuron)

- sympathische Systemaktivierung, parasympathische Dysregulation

- zentrale Sensibilisierung (spinaler "crosstalk“, „primping"), neurogene Neuroinflammation

- Aktivierung rezeptiver Felder, „referred pain“, „wide spread pain“

- sekundäre Hyperalgesie

- Dysregulation schmerzinhibitorischer Systeme

- zentrale Chronifizierung, Schmerzkrankheit

Da die neurogene Inflammation, als relativ früher einsetzender Mechanismus der peripheren Sensibilisierung, auf isolierte MMInterventionen nicht anspricht, dienen primär antiinflammatorische Analgetika als spezifische Therapeutika. 
Tab. 1 Vertebragene Nozizeptoren

\begin{tabular}{|l|l|l|}
\hline Noziregion & Physiologisch & Pathologisch \\
\hline Ossär & $\begin{array}{l}\text { Rr. communicantes Nn. spinales } \\
\text { Gefäße (vegetativ, sympathisch) }\end{array}$ & $\begin{array}{l}\text { Subkortikale Hypervaskularisation } \\
\text { bei Diskopathie }\end{array}$ \\
\hline Ligamentär & $\begin{array}{l}\text { Ligg. ileolumbale, Ligg. longitudina- } \\
\text { le etc. } \\
\text { Intraspinal: z. B. Ligg. flava }\end{array}$ & $\begin{array}{l}\text { Überdehnung, Zerrung } \\
\text { Enthesiopathie, Fasziitis } \\
\text { Hypertrophie }\end{array}$ \\
\hline Diskogen & Anulus fibrosus & $\begin{array}{l}\text { Diskopathie/Diszitis: Neovaskularisa- } \\
\text { tion des N. pulposus }\end{array}$ \\
\hline Neural (Dura) & Partiell & Neuritis \\
\hline SIG) & $\begin{array}{l}\text { Gelenkkapsel } \\
\text { Mit Meniskoiden (bei }>50 \% \text { vor- } \\
\text { handen), partiell vaskularisiert und } \\
\text { innerviert }\end{array}$ & $\begin{array}{l}\text { Arthritis, Kapsulitis, Synovialitis } \\
\text { Einklemmung von Meniskoiden: } \\
\text { Einblutung, Degeneration }\end{array}$ \\
\hline Myofaszial & Myofaszialer Nervenfaserverlauf & $\begin{array}{l}\text { Propriozeptive Rückkopplung: Mus- } \\
\text { kelhypertonus/Myalgie } \\
\text { Myositis, Fasziitis }\end{array}$ \\
\hline FG Facettengelenk, Ligg. Ligamente, SIG Kreuzdarmbeingelenk \\
\hline
\end{tabular}

Injektable analgetischantiinflammatorische Agenzien

Therapeutische Lokalanästhesie (TLA) unter Verwendung niedrigdosierter Lokalanästhetika (LA) werden seit mehr als 10 Dekaden zur Reduktion der Nervenerregbarkeit bzw. Schmerzen (Blockade sensibilisierter Nervenfasern) und Verbesserung der Durchblutung eingesetzt [48]. Unter anderem werden dabei die dünnen sensiblen Schmerzfasern (C-Fasern und A $\delta$-Fasern) weitgehend blockiert, wobei die Erregbarkeit der dickeren motorischen Fasern i.d. R. erhalten bleibt. Mechanismen der desensibilisierenden Wirkung der LA sind direkt anti-inflammatorische LA-Effekte und die Reduktion der "neurogenen Inflammation" durch verminderte Ausschüttung von Neuropeptiden (z. B. CGRP, Substanz P), welche die Prostaglandin- und Zytokinbildung (z. B. TNF-a, IL6, IL-1ß) fördern [13]. Additiv beigefügte Glukokortikoide (GK) können den pathologischen Nozizeptorschmerz aufgrund des inflammatorisch gereizten Gewebes (neurogene Inflammation), sowie auch einen neuropathischen Schmerz (inflammatorisch gereizte Nervenfasern), d.h. auch den „mixed pain“, effektiv down-regulieren [4]. Durch Reduktion der Synthese von Interleukinen und Phospholipase A2 und durch Vasoprotektion wirken GK hemmend auf den, v.a. durch cytokinproduzierende Zellen ausgelösten, biochemischen Entzündungsprozess bei der Radikulitis ein: Hemmung von Migration bzw. Ausdifferenzierung von Entzündungszellen zu
Makrophagen [67]: TLA-Wiederholungen verbessern den Wirkeffekt bzw. wirken durch eine verlängerte Desensibilisierung der Schmerzchronifizierung entgegen [46].

\section{Initialdiagnostik}

Die First-Line-Diagnostik bei TRS umfasst neben der allgemeinen und vegetativen (Fremd-)Anamnese die spezifische Schmerzanamnese, welche die Evaluierung von Schmerzlokalisation, -qualität, -quantität (VAS/NRS), -häufigkeit, -dauer sowie der internen Trigger- und Risikofaktoren ("red flags" [102]) umfassen sollte. Bei V.a. chronischen TRS ist die validierte Evaluation bzgl. Chronifizierungsstadium (MPSS [32]) und neuropathischer Schmerzkomponente („pain detect" [31]), wie auch die anamnestische Erfassung psychosozialer beruflicher (,blue/black flags“" [42]) und soziofamiliärer („yellow flags“ [11]) Risikofaktoren der Schmerzchronifizierung Standard.

\section{॥ Die physikalische Untersuchung ist das zentrale Element der diagnostischen Bemühungen}

Die physikalische Untersuchung als zentrales Element der diagnostischen Bemühungen umfasst dabei klassischerweise die Inspektion von Gang, Achssymmetrie, Haltung etc., die Palpation hinsichtlich Dermographismus, vegetative Zeichen, Gewebeturgor etc., und die funktionelle akti- ve/passive Bewegungsprüfung (ROM) mit spezifischen Funktionstests. Orientierende neurologische Untersuchung hinsichtlich Durchblutung, Motorik und Sensibilität, Reflexstatus, Nervendehnungs-/-provokationszeichen etc. sind inbegriffen.

Additiv ist für den manualmedizinisch tätigen Arzt die segmentale nozizeptive Funktionsanalyse mittels 3-Schritt-Diagnostik (MIP) zur segmentalen Evaluierung von Mobilität (segmental), Irritation (nozireaktiver Muskelhypertonus) und Provokation (Reversibilität?) zentrales und einziges gut reliables, europäisch konsentiertes und definiertes, manualdiagnostisches Instrument $[92,101]$ zum Einstieg in mechanismenbasierte Überlegungen.

\section{Bildgebung}

Bei akutem TRS (unter 6 Wochen Schmerzdauer nach Erstauftreten) ist eine Bildgebung dann sicher indiziert, wenn aufgrund Anamnese und klinischer Untersuchung Hinweise für einen spezifischen Rückenschmerz oder "red flags" bestehen [11]. Laut Leitlinie ist für die Diagnose eines spezifischen Kreuzschmerzes i.d. R. eine bildgebende Diagnostik notwendig [34]. Eine wiederholte analgetisch-antiphlogistische Medikation und/oder MM-Therapie muss also nach Verstreichen der Akutphase der TRS bei ausbleibender Beschwerdelinderung hinsichtlich deren Ursache spezifiziert bzw. bildgebend abgeklärt werden. Die Basisdiagnostik besteht aus einem konventionellen Röntgenbild der (LWS im Stand in 2 Ebenen) sowie einer Magnetresonanztomographie (MRT) der LWS [34], eventuell auch der Sakroiliakalgelenke (SIG), unter Berücksichtigung des $\S 23$ RöV und der Leitlinien der Bundesärztekammer (BÄK) zur Qualitätssicherung in der Röntgendiagnostik [50]. Die Diskussion, ob Röntgen oder MRT, ist nicht neu und richtet sich nach der Aussagefähigkeit der Untersuchung im Hinblick auf die Verdachtsdiagnose [86], aber initial auch nach der Verfügbarkeit, der Frage der Strahlenexposition und der Kosten [11]. Daher wird die Frage einer notwendigen Computertomographie (CT) der LSIR nur bei traumatischen oder präoperativen Fragestellungen relevant [86]. Bezogen auf die MM in der Akutphase von TRS, ist nach Durchführung einer Probemobilisation vor einer 
Tab. 2 Klinische Schmerzmuster beim TRS, modifiziert nach Heisel und Jerosch [37]

\begin{tabular}{|c|c|c|}
\hline Schmerzmuster & Schmerzcharakteristik (Symptomatik/Klinik) & $\begin{array}{l}\text { Neurophysiologische } \\
\text { Schmerzmechanismen }\end{array}$ \\
\hline Lokaler Schmerz & $\begin{array}{l}\text { Fokale somatische Schmerzen über dem } \\
\text { Schmerzgenerator, evtl. mit Hyperalgesie/- } \\
\text { ästhesie } \\
\text { z. B. Arthritis/aktivierte Arthrose über FG/SIG; } \\
\text { Enthesiopathie: z. B. SIPS, Myalgie (z. B. M. lum- } \\
\text { borum) }\end{array}$ & $\begin{array}{l}\text { Primäre Hyperalgesie: } \\
\text { akut, nozizeptiv } \\
\text { neurogene Inflammation }\end{array}$ \\
\hline $\begin{array}{l}\text { Übertragener } \\
\text { Schmerz („re- } \\
\text { ferred pain“) }\end{array}$ & $\begin{array}{l}\text { Schmerzprojektion aus tiefen viszeralen (o. so- } \\
\text { matischen) Strukturen auf ein konstantes } \\
\text { Segment } \\
\text { "Stechender“, durchdringend, brennender u./o. } \\
\text { temperaturempfindlicher Schmerz } \\
\text { z. B. Hyperalgetische Zonen: Head-Zonen } \\
\text { (Haut) oder McKenzie-Zonen (Muskeln) } \\
\text { z. B. Muskelhypertonie, autonome Veränderun- } \\
\text { gen (keine Reflexalteration/Muskelatrophie) }\end{array}$ & $\begin{array}{l}\text { Afferente Konvergenz aus } \\
\text { Neurotom-Viszerotom- } \\
\text { Myotom-Dermatom auf } \\
\text { die Hinterhornzelle (WDR) }\end{array}$ \\
\hline $\begin{array}{l}\text { Myofasziale } \\
\text { Triggerpunkte }\end{array}$ & $\begin{array}{l}\text { Schmerzprojektion auf nahe bis überregio- } \\
\text { nale Areale in tiefe anatomische Schichten } \\
\text { (myofaszial) } \\
\text { "Nackter-einfacher" Schmerz } \\
\text { z. B. pseudoradikulärer Schmerz als Ausdruck } \\
\text { artikulärer Störung mit reflektorischer Muske- } \\
\text { lirritation (wirbelsäulennaher Schmerzgenera- } \\
\text { tor) }\end{array}$ & $\begin{array}{l}\text { Langsame zentrale Sensi- } \\
\text { bilisierung und Ausbrei- } \\
\text { tung auf rezeptive Felder } \\
\text { [63] } \\
\text { sekundäre Hyperalgesie } \\
\text { (neuropathisches Gesche- } \\
\text { hen), Senkung Schmerz- } \\
\text { schwelle }\end{array}$ \\
\hline $\begin{array}{l}\text { Ausstrahlender, } \\
\text { fortgeleiteter } \\
\text { Schmerz }\end{array}$ & $\begin{array}{l}\text { Vor allem im Ausbreitungsgebiet neuraler } \\
\text { Strukturen (Dermatom, Myotom, Sklerotom) } \\
\text { z.B. Neuralgie, Plexusneuritis, Radikulopathie }\end{array}$ & $\begin{array}{l}\text { Neuritis (neurale Inflam- } \\
\text { mation) }\end{array}$ \\
\hline
\end{tabular}

Manipulation ein vorheriges routinemäßiges LWS-Röntgen nicht notwendig [11, 43].

\section{3-Ebenen-Diagnose}

In der Regel sollte zu diesem Zeitpunkt eine erste mechanismenbasierte Funktionsdiagnose angestrebt werden. Hier hat sich in den letzten Jahren in der MM (DGMM) die Formulierung der sog. 3-Ebenen-Diagnose als praktikables und sinnvolles Tool erwiesen [52], welches auf der ersten Ebene $A$ die Symptombeschreibung der Beschwerden des Patienten und dessen subjektives Erleben, sowie die zeitlichen (Akuität, Chronizität) und räumlichen Faktoren (Lokalität) beinhaltet. Die zweite Ebene B umfasst die Beschreibung der möglichen somatischen Genese der Beschwerden, einschließlich struktureller und funktioneller Befunde, sowie, falls bereits möglich, die mechanistische Genese mit Fokus auf den neurophysiologisch-mechanistischen Faktoren. Zuletzt folgt Ebene $\mathrm{C}$ mit der Berücksichtigung der soziofamiliären Situation des Patienten (Beruf, Familie, Ökonomie).

\section{Schmerzmuster}

Aus dem Schmerzmuster („pain mapping ") kutanomyofaszialer Schmerzen, welches sich bei Patienten mit TRS im Rahmen der klinisch-palpatorischen Diagnostik evaluieren lässt, kann der erfahrene Untersucher wertvolle Rückschlüsse hinsichtlich der Schmerzgenese ziehen [37, 63, 103]. Entscheidend ist hierbei, die fokalen Schmerzen und myofaszialen Triggerpunkte von ausstrahlenden, fortgeleiteten und übertragenen Schmerzen richtig zu differenzieren und einzuordnen (- Tab. 2).

\section{Kasuistik-Teil 1: Klinische Kasuistik}

Ein 55-jähriger Mann, der sich initial mit seit 3 Tagen exazerbierten TRS (lumbal bilateral gluteal bis in den dorsolateraleren Oberschenkel rechts reichend) mit einer Schmerzstärke von 9 (10-Pkt.-VAS) vorstellt.

\section{Anamnese}

Seit ca. 12 Monaten bestehender „stechend-ziehender" Schmerz in der rechts- dominanten $L S I R$, rezidivierend bis zur laterodorsalen Unterschenkelregion reichend. Hierbei nur kurze schmerzfreie Intervalle über 1-3 Tage bei einer Schmerzdauer von mehreren Tagen bis Wochen. Belastungsabhängige Schmerzverstärkung am Tage, bewegungs- und lagerungsabhängiger Schmerz in Ruhe und zur Nacht und v.a. morgens beim Aufstehen.

Nebenerkrankungen: Diabetes mellitus Typ Ilb (Rp Metformin), arterielle Hypertonie (Rp Ramipril), Z.n. Koronarstents bei 2-Gefäß-KHK, Hypercholesterinämie (Rp Statin), Adipositas (BMI $33 \mathrm{~kg} / \mathrm{m}^{2}$ ), Obstipationsneigung mit gelegentlich Unterbauchschmerzen.

Sozialanamnese: Identifikation mit und Freude am Beruf als Altenkrankenpfleger (2-Schicht-System mit regelhaften Transferarbeiten). Soziofamilär seit Jahren zufrieden verheiratet, 2 erwachsene Kinder (außer Haus). Tennis als Hobby, seit Monaten schmerzbedingt pausiert.

\section{Klinischer Erstbefund}

Schmerzbedingte linksorientierte Rumpfschiefhaltung mit Entlordosierung der LWS, deutlicher Reduktion des FingerBoden-Abstands und LWS-Entfaltungsstörung (Reduktion Schober/Ott). Segmentale Funktionsanalyse (MIP) schmerzbedingt kaum möglich bzw. nicht interpretierbar. Orientierend neurologisch unauffällige Sensibilität, (Pseudo-)Lasègue ab $60^{\circ}$ links, beidseits symmetrische Hyporeflexie bzgl. Achillessehnenreflex/ Patellarsehnenreflex. Palpatorisches Schmerzmuster mit ausgeprägter Berührungsempfindlichkeit der gesamten lumbopelvinen Region bis gluteal und starke Druckempfindlichkeit lumbosakral bilateral paravertebral (L3-S2) und über den SIPS und proximale SIG bis gluteal links (Valleix-Punkt links positiv). Die Betrachtung der entsprechenden „pain map“ in - Abb. 1a und b führt differenzialdiagnostisch nicht sicher weiter, da vereinbar mit (- Tab. 2):

- lokalem Schmerz bei Facettensyndrom (FGS) L5/S1 (oder L4/5) [73, 108] bei Spondylarthrose,

- viszerogen übertragenem Schmerz („referred pain“): z. B. aus Dickdarm (Th11), bei bekannten Unterbauchschmerzen [103], 


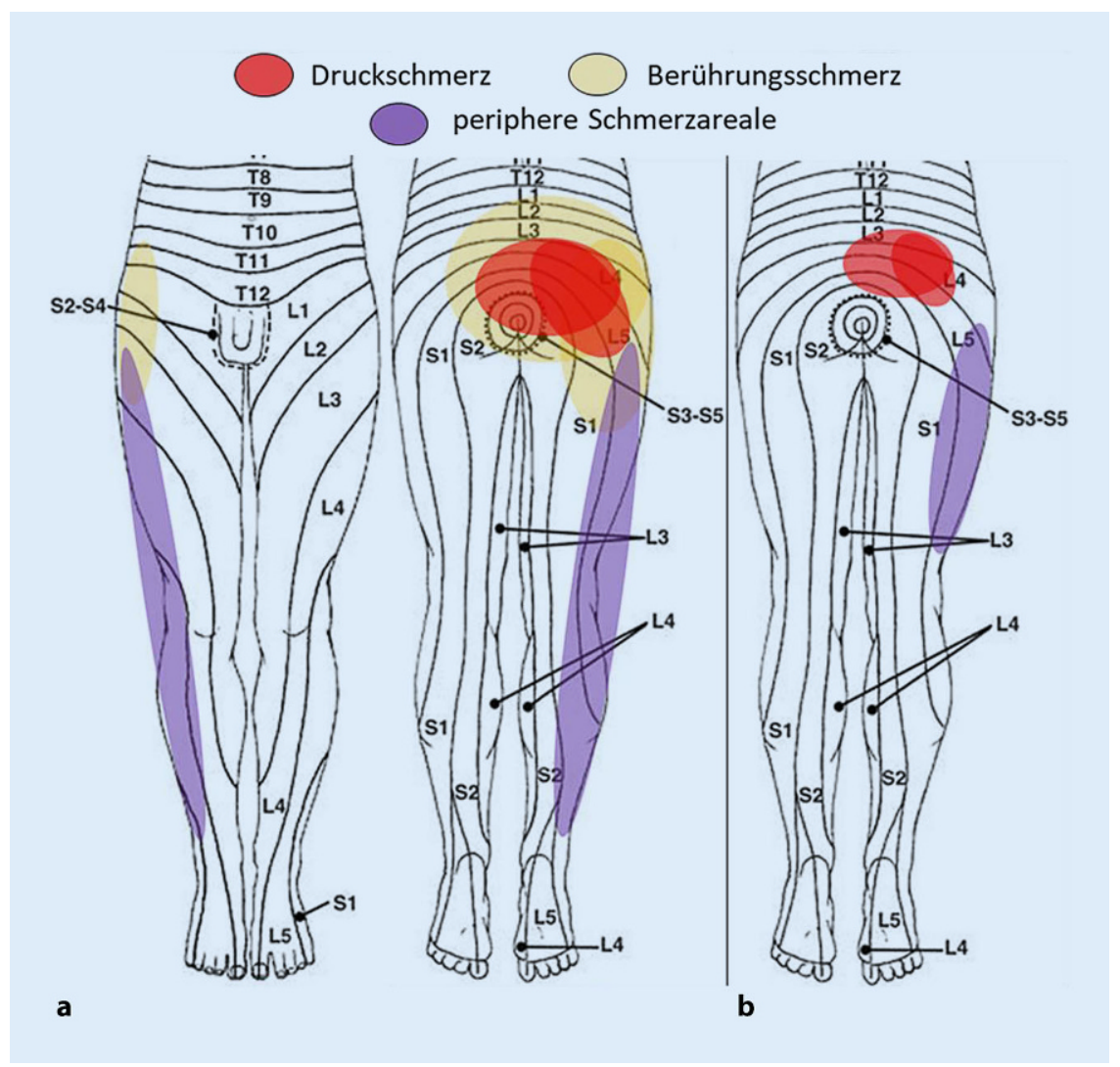

Abb. 1 ॥ Kasuistik -Schmerzbild („pain map“), a bei Erstvorstellung, b bei Re-Assessment nach 14 tagen; (modifiziert [109])

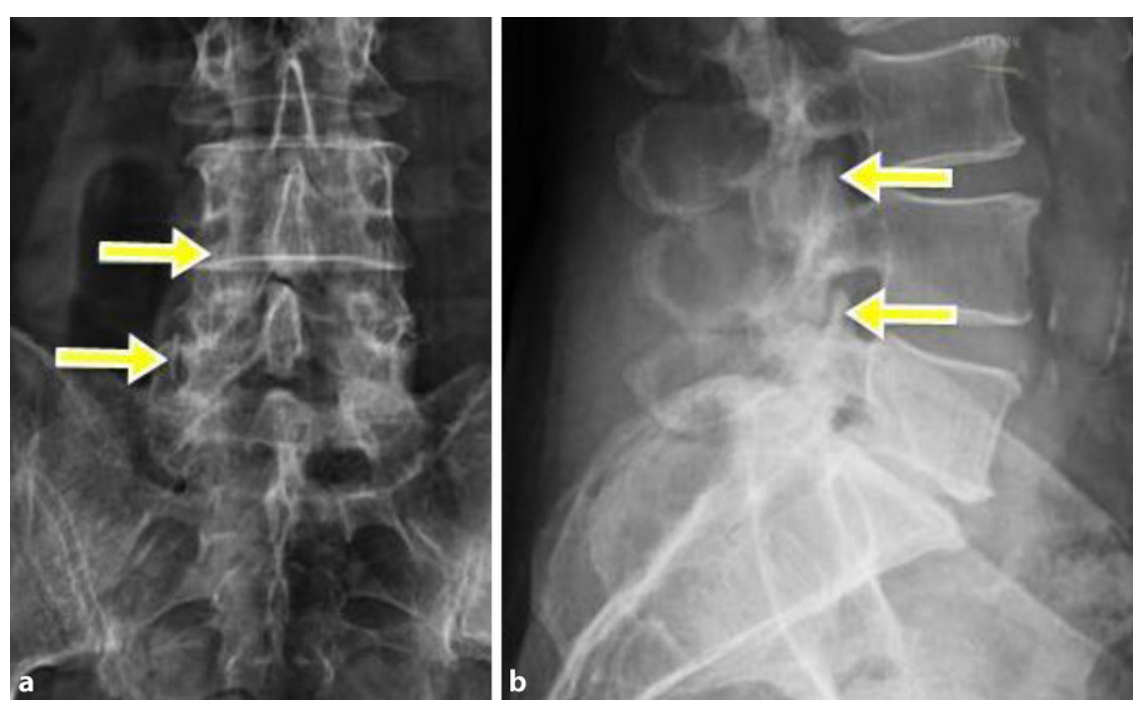

Abb. 2 \ Kasuistik - Übersichtradiographie Lendenwirbelsäule in 2 Ebenen (a anterioposterior, b seitlich). Pfeile Facettengelenke L3/4 und L4/5 rechts. (Mit freundl. Genehmigung, $\odot$ U. Schütz, alle Rechte vorbehalten.)
- pseudoradikulärem Schmerz als Ausdruck artikulärer Störung mit reflektorischer Muskelirritation (manifestiert als Triggerpunkte) von M. iliocostalis/ longissimus [28], M. quadratus lumborum oder M. gluteus medius [24], und/oder

- radikulärem Schmerz entsprechend Dermatom L5/S1 rechts, bei Minderung des Intervertebralraumes (IVR) L5/S1.

Röntgen LWS in 2 Ebenen ( $\mathbf{0}$ Abb. 2) Orthogerader Aufbau (a.-p.) und Entlordosierung (Seitprojektion), Osteochondrose L5/S1 (Grad 3-4) bei Diskopathie (IVR-Höhenminderung $70-90 \%)$ und Spondylarthrose L5/S1 (Grad 2-3) und L4/5 (Grad 2). Partiell abgebildete kaudale SIG-Arthrose links (Grad 2-3).

\section{Procedere}

Bei dem Patienten findet sich übersichtradiographisch eine fortgeschrittene IVRDegeneration als Hinweis auf eine relevante Diskopathie, klinisch ist eine biomechanisch bedingte Wurzelirritation L5/S1 links möglich. Dies bedingt aufgrund der Ausprägung der Beschwerdesymptomatik die weiterführende Abklärung mittels MRT der LWS und Einholung einer neuro(physio)logischen Mitbeurteilung.

\section{Multiple Schmerzgeneratoren bei degenerativem TRS}

Eine progrediente IVR-Degeneration führt bei Verlaufsprogredienz biomechanisch, pathophysiologisch bedingt zu multilokulären Veränderungen mit möglicher Veränderung und/oder Verschlimmerung der Symptomatik, auch über das Segment hinaus.

Bei der alters- bzw. be-/überlastungsbedingten Bandscheibendegeneration, welche i. d. R. im Bereich L3-S1 aufsteigend initiiert wird, kommt es durch Dehydrierung des Bandscheibengewebes u. a. zu kleinen Einrissen mit Strukturschwäche des Anulus fibrosus und konsekutiver Vorwölbung, welche favorisiert dorsal betont imponiert und hier auch fokal zum Riss des Anulus fibrosus mit Austritt von Nucleuspulposus-Material führen kann. Diese Zeichen der degenerativen Diskopathie sind nur mittels MRT der LWS direkt darstellbar („,black disc“, Protrusion, Prolaps) [86]. 


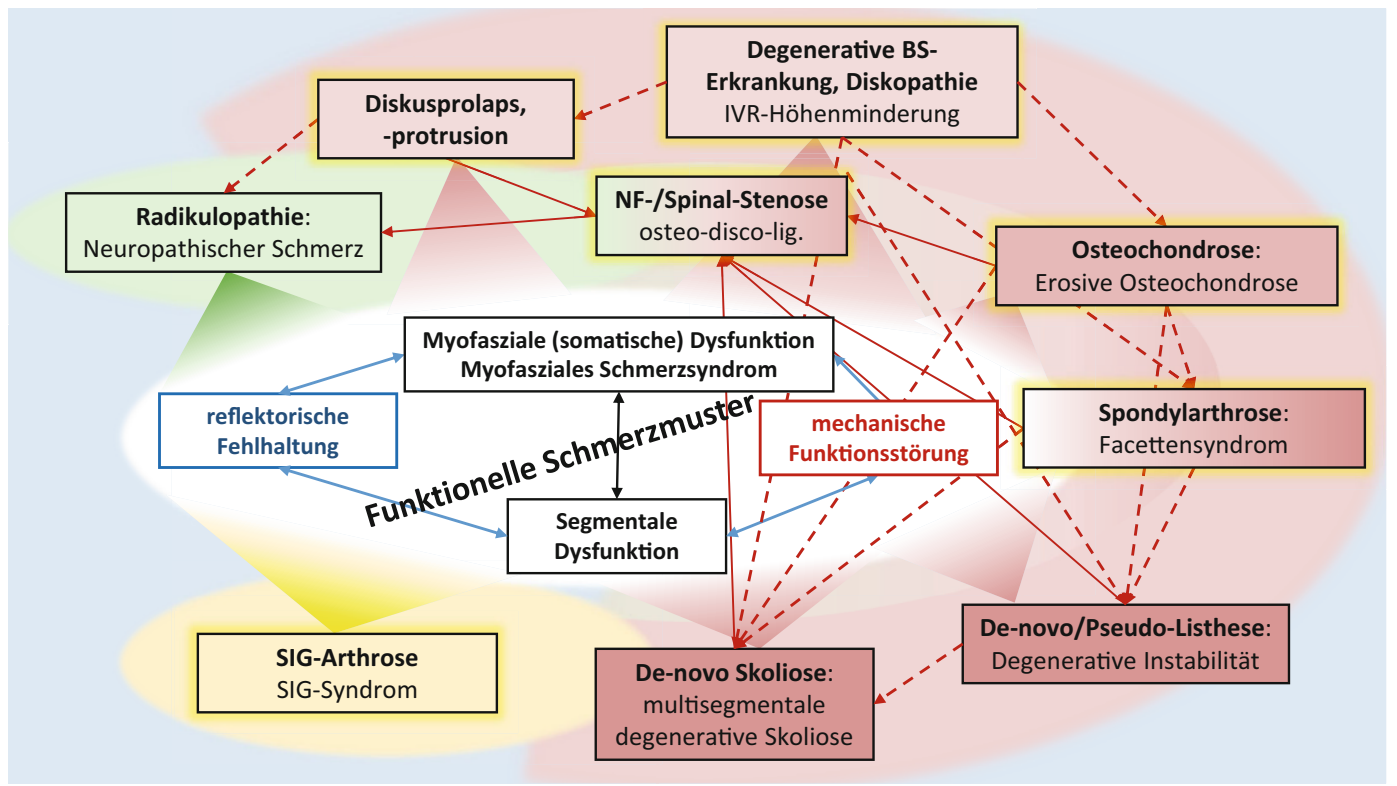

Abb. 3 ॥ Strukturelles und funktionelles pathophysiologisches Bedingungsgefüge bei degenerativem tiefem Rückenschmerz. Weiß Funktionelle primäre oder sekundäre Schmerzmuster, Rot Mögliche strukturelle Pathologien im Bereich der Lendenwirbelsäulensegmente, welche primär ( gelb umrandet) und/oder sekundär als Folgereaktion auftreten können und direkt mechanische Funktionsstörungen bedingen können, und auch sekundäre funktionelle Störungsmuster generieren, Grün Radikulopathie, neuropathischer Schmerz als Folge degenerativer Strukturprozesse und wichtiger Generator funktioneller Schmerzmuster. Gelb umrandet primäre Schmerzgeneratoren aufgrund degenerativer Strukturpathologie, BS Bandscheibe, IVR Intervertebralraum, lig. ligamentär, NF Neuroforamen, SIG Sakroiliakalgelenk (Mit freundl. Genehmigung, $\odot$ U. Schütz, alle Rechte vorbehalten.)

Mit zunehmender Höhenminderung der Bandscheibe kommt es zur Annäherung der entsprechenden Wirbelkörper (röntgenologisch indirekt durch die Reduktion der IVR-Höhe detektierbar: (hondrose), was auch zu einer Erhöhung der Druckbelastung der angrenzenden Boden- bzw. Deckplatten führt, welche mit der Zeit zur Strukturveränderung der Kortikalis führt (Röntgen: Sklerose). In der Progredienz kommt es am IVR zur Bildung ossäre Abstützreaktionen (Röntgen/MRT: Spondylophyten), welche regelhaft anterolateral, aber auch dorsal auftreten können. Im Verlauf kommt es zu einem fettig-degenerativen Umbau (MRT: Typ Modic 2) und/oder Dehydrierung des angrenzenden Markraumes (MRT: Typ Modic 3). Bei diesem Bild der Osteochondrose findet sich, als i.d.R. stärker beschwerdebehaftetes Zwischenstadium, eine entzündliche Überlastungsreaktion des angrenzenden spongiösen Markraumes mit primär intrakorporaler Ödembildung (MRT: Typ Modic 1), welches dann als erosive Osteochondrose bezeichnet wird.
Die IVR-Höhenminderung führt auch zu vermehrten chondralen Druckbelastungen in den Facettengelenken (FG). Hierdurch wird ein Arthroseprozess initiiert, wie er auch pathophysiologisch an peripheren Gelenken auftritt [85]. Es entsteht eine Spondylarthrose, welche in Progredienz typische bildgebende Arthrosezeichen entwickelt: Inkongruenz der Gelenkpartner, FG-Hypertrophie durch Osteophytosis, Ergussbildung. So kommt es auch bei weiterer Progredienz von Degeneration und Höhenminderung des IVR zur Deformierung von angrenzenden Deckund Bodenplatten bzw. Wirbelkörperanteilen, auch mit partieller Gefügelockerung, was eine segmentale Stellungsveränderung der Wirbelkörper bedingen kann, welche entweder als Retro- oder Pseudolisthese oder/und multisegmental zu eine sog. De-novo-Skoliose der LWS führen kann.

Retrospondylophytosis, Verkalkung des hinteren Längsbandes, reaktive Hypertrophie der Ligg. flava, Listhese, Skoliose und/ oder Diskusprotrusion/-prolaps können eine segmentale Stenosierung neuraler Räume bedingen, welche dann in der MRT als spinale, rezessuale und/oder intraforaminale Stenosen auf osteo-, disko- und/oder ligamentärer Basis zu beschreiben sind und als Radikulopathie und/oder Claudicatio spinalis im Sinne des neuropathischen Schmerzes bzw. Geschehens symptomatisch werden.

Die beschriebene Strukturpathogenese progredienter degenerativer Segmentveränderungen, welche allesamt mittels MRT (Goldstandard beim TRS) detektierbar sind [86], werden in ihrem komplexen gegenseitigen Bedingungsgefüge in - Abb. 3 (roter Bereich) grob schematisch aufgezeigt.

Unter Kenntnis der möglichen Schmerzgeneratoren ( $\boldsymbol{0}$ Tab. 1) und der Neurophysiologie der Schmerzentstehung und -weiterleitung [52, 66, 75, 81], können alle in - Abb. 3 aufgezeigten Strukturpathologien (rot-grün-gelber „Ring ${ }^{\prime \prime}$ ) primärer Schmerzgenerator bei degenerativem TRS sein. Wie aufgezeigt, kann jeder dieser primären Schmerzgeneratoren schmerzreflektorisch (segmentale Nozireaktion) funktionelle Schmerzmuster (0 Abb. 3: zentral weiß hervorgehoben) verursachen, welche klinisch als myofasziales 
Tab. 3 Effektivität und Evidenz der Manuellen Therapie beim TRS, tabellarischer Literaturreview

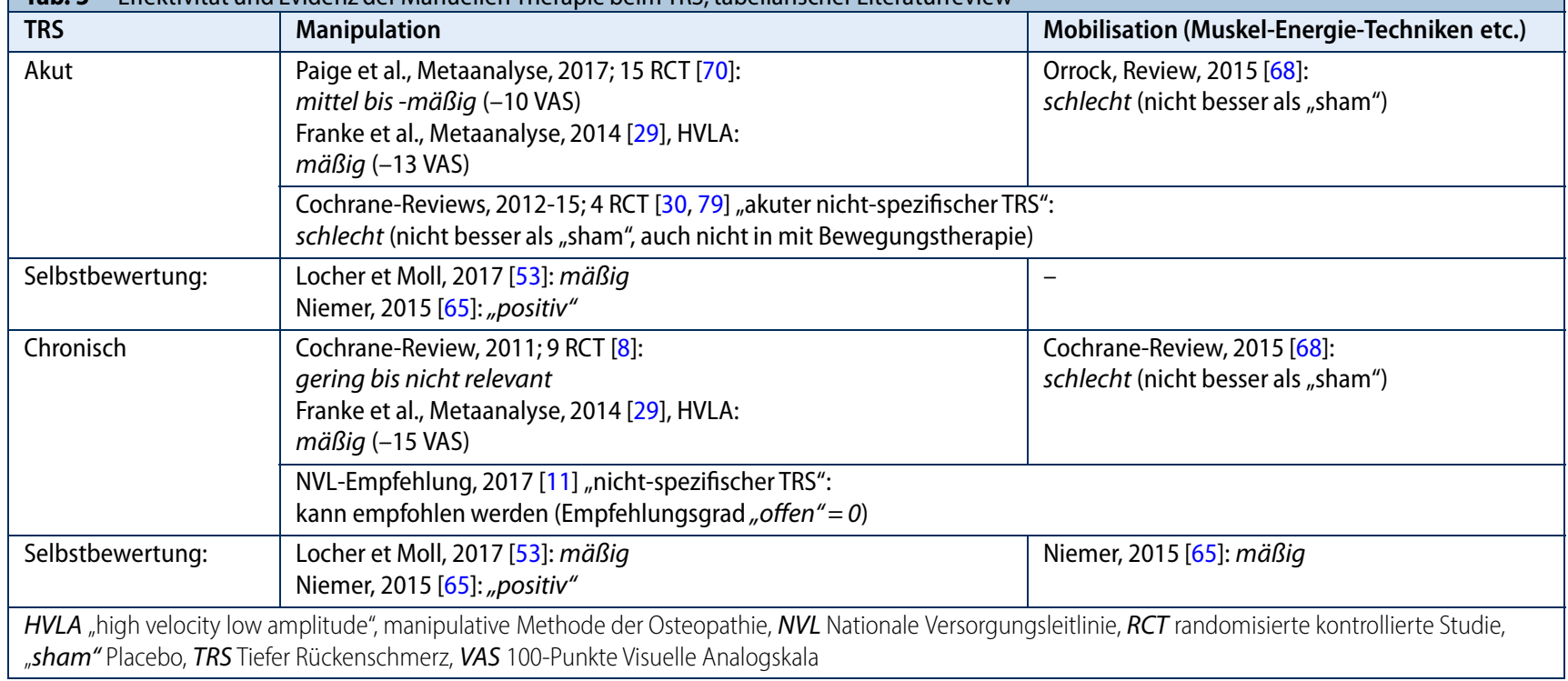

Schmerzsyndrom/Dysfunktion und/oder hypomobile segmentale Dysfunktion (Blockierung) zum Ausdruck kommen.

\section{Manuelle Medizin bei TRS: Definition, Indikation, Evidenz}

Eine einheitliche Definition der Manuellen Medizin gibt es nicht. Laut (Muster-)Weiterbildungsordnung der BÄK [107] umfasst

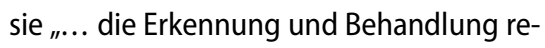
versibler Funktionsstörungen des Bewegungssystems einschließlich ihrer Wechselwirkung mit anderen Organsystemen mittels manueller Untersuchungs- und Behandlungstechniken."

Im Kern bedient sich die MM der Manipulation und der Mobilisation. Erstere sind Techniken mit Impuls und werden im Bereich der Osteopathie als HVLATechniken (,high velocitiy low amplitude") bezeichnet und haben beim TRS ihre Indikation bei reversiblen, hypomobilen Funktionsstörungen ohne Radikulopathie (= Blockierung) in der LSIR [11,51], welche leitlinienkonform Ursache spezifischer TRS sein kann [34]. Techniken der Mobilisation umfassen beim TRS u. a. wiederholt, rhythmisch-federnde Traktions-TranslationsBewegungen, z. B. Muskel-Energie-Techniken, und im osteopathischen Bereich auch Strain-/Counterstrain, Myofascial Release etc., und haben ihre Indikationen in allen reflektorischen und teils strukturellen Dysfunktions- und Schmerzzustände der LSIR mit (pseudo-)radikulärer Ausstrah- lung verschiedenster Genese [11, 51], zu der v.a. das myofasziale Lumbalsyndrom (Dysfunktion) als Ursache spezifischen TRS [34] zählt. Hinsichtlich der schmerzreduzierenden Wirkungsweise der MM wird auf die einschlägige Fachliteratur [51, 54] und auf den Beitrag von H. Schnell et al. in diesem Themenheft („Die segmentale und somatische Dysfunktion. Wie funktioniert Manuelle Medizin?") verwiesen.

\section{Evidenz der MM}

Wie aus - Tab. 3 ersichtlich, ist bei Anlegung von evidenzbasierten Kriterien die Effektivität für die Manipulation beim akuten und chronischen TRS nur mäßig, für die Mobilisationstechniken eher sogar schlecht. Lediglich eine Selbstbewertung kommt zu einer positiveren Bewertung, welche keiner Evidenzkontrolle standhält [65], zumal selbst führende Kapazitäten auf dem Gebiet der MM deren Evidenzlage als nur mäßig [53] bewerten.

Betrachtet man obige Ausführungen zu den vielfältigen Möglichkeiten der primären Schmerzgenerierung beim TRS und dessen strukturpathologisches Bedingungsgefüge bis hin zur regelhaften Folgegenerierung funktioneller Schmerzmuster (『Abb. 3), so gibt dies einen Hinweis, dass ein myofasziales Lumbalsyndrom und eine Blockierung in Gegenwart degenerativer Veränderungen zu einem hohen Anteil sekundärer Natur und nicht regelhaft primäre Entitäten sind. In der
Schlussfolgerung ist dann die schlechte Evidenzlage der Therapiewirkung von MM auch mit dadurch erklärbar, da oft der primäre Schmerzgenerator (Strukturpathologie) und damit der Verursacher funktioneller Störmuster bei Therapie noch gar nicht identifiziert bzw. eliminiert ist, was konsekutiv Rezidive von myofaszialer und hypomobiler somatischer Dysfunktionen in der LSIR trotz wiederholter MMInterventionen erklärt.

Insofern muss jeder MM-Therapeut bei, trotz lege artis durchgeführter MM-Techniken, rezidivierend auftretenden funktionellen Schmerzmustern ein kritisches ReAssessment durchführen und prüfen, ob die Diagnostik hinsichtlich der Detektierung des primären Schmerzgenerators abgeschlossen oder auszuweiten ist, bzw. sich fragen, ob er den Patienten in seinem TRS mechanismenbasiert korrekt erfasst hat.

\section{Kasuistik-Teil 2: 3-Ebenen-Diagnose und Erstbehandlung}

Nach Erstdiagnostik wird folgende Funktionsdiagnose gestellt:

Ebene A: Exazerbiertes rechtsdominantes Schmerzsyndrom der LSIR bei chronischer belastungs- und bewegungsabhängiger Lumbosakralgie mit rezidivierendem Beinschmerz rechts dorsolateral. 


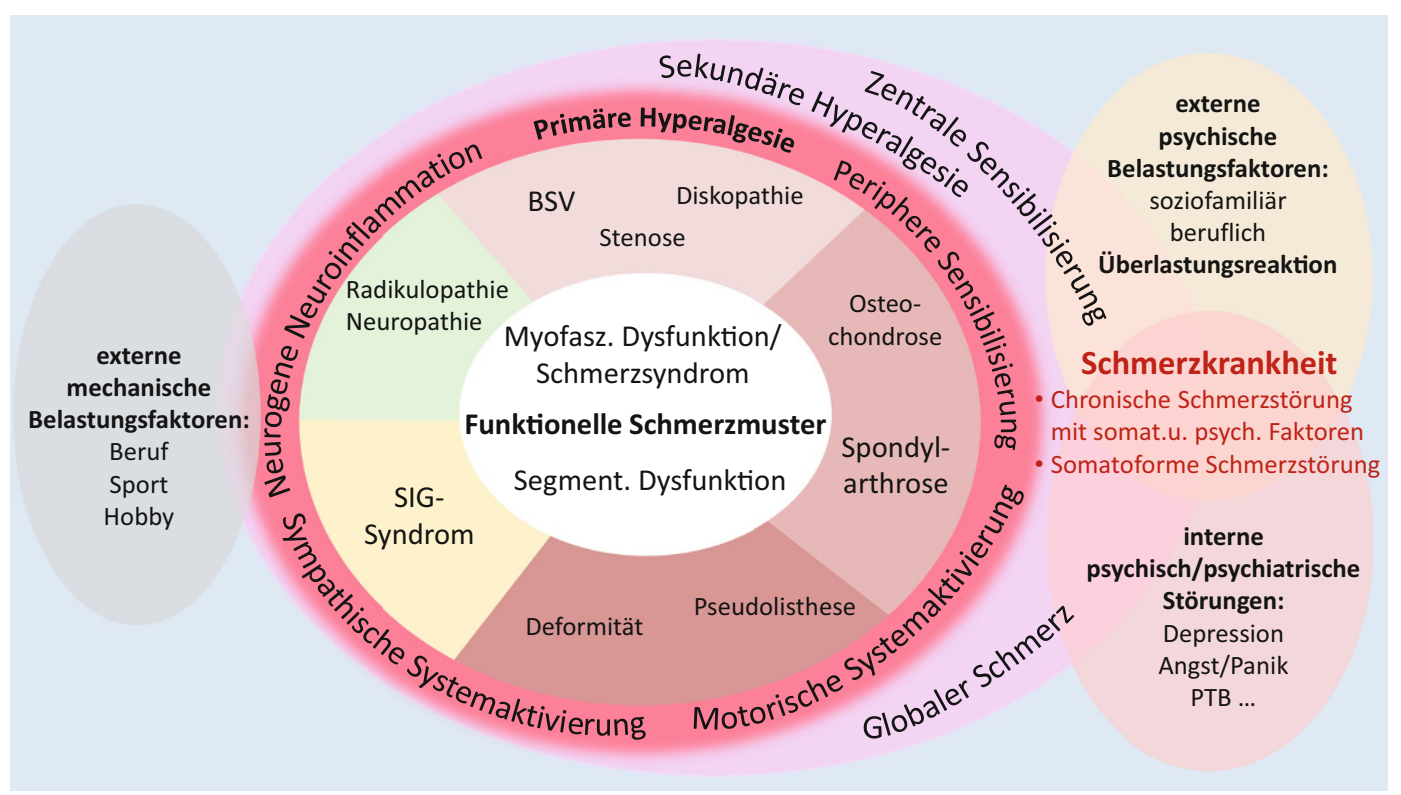

Abb. $4 \triangleleft$ Mechanismenbasiertes Bedingungsgefüge vom einfachen nozizeptiven bis hin zum chronifizierten tiefen Rückenschmerz. BSV Bandscheibenvorfall. SIG Sakroiliakalgelenk, PTB Posttraumatische Belastungsstörung (Mit freundl. Genehmigung, $\odot$ U. Schütz, alle Rechte vorbehalten.)
Ebene B:

- chronisches (sekundäres) myofasziales Schmerzsyndrom mit segmentaler Funktionsstörung (Blockierung)

- Strukturpathologien: Osteochondrose L5/S1, Spondylarthrose L4-S1, SIGArthrose links

- DD Schmerzmuster: (Pseudo-)Radikulopathie, „referred“ (viszeral: Colon?), lokal (FG?, SIG?)

- exazerbierter Schmerz (neurogene Neuroinflammation, primäre Hyperalgesie, motorische Systemaktivierung, periphere Sensibilisierung)

- chronischer Schmerz, „wide spread pain" (sekundäre Hyperalgesie, zentrale Sensibilisierung)

\section{Ebene C:}

- „black flag": Transferarbeiten. Freude am Beruf (Ausschluss „blue flags“)

- soziofamiliär, psychogen keine Auffälligkeiten (Ausschluss „yellow flags“)

Aktuell ist eine erneute MM-Therapie aufgrund der Schmerzexazerbierung nicht durchführbar und auch nicht indiziert, da sie bisher nur sehr kurzfristig zur Besserung betrug. Die Chronifizierung lässt vermuten, dass, bei vorliegender primärer Hyperalgesie bei peripherer Sensibilisierung, neurogener Neuroinflammation sowie sympathischer und motorischer Systemaktivierung, der Übergang zur sekundären Hyperalgesie mit zentraler Sensibilisierung und damit zum zentralen Chronifizierungsprozess bereits begonnen hat - auch wenn bei dem Patienten „nur" externe mechanische Belastungsfaktoren im Beruf („,black flags") und keine externen psychischen soziofamiliären („yellow flags") oder beruflichen („,blue flags ") und auch keine intrinsischen psychisch/psychiatrischen Störungen vorliegen (•Abb.4).

In der vorliegenden klinischen Situation hat die rasche und wirksame Schmerzreduktion oberste Priorität, um eine klinische Feindiagnostik und auch die Therapiefähigkeit bezüglich konservativ multimodaler Interventionen zu ermöglichen. Nach dem Prinzip „hithard and early“ ist hier eine Methodik zu wählen, die rasch und effektiv ist, sodass der Kaudalblock eine Möglichkeit darstellt, um dieses Ziel zu erreichen. Bei Beherrschung und korrekter technischer Durchführung dieser Technik, bietet dieser gegenüber der potenten Schmerzinfusion den Vorteil der guten Verträglichkeit ohne relevante systemische Belastung.

Beim Patienten wurde bei Erstvorstellung ein landmarkengestützter Kaudalblock (Gemisch: Triamcinolon $20 \mathrm{mg}$ Off Label, $15 \mathrm{ml}$ Ropivacain $2 \mathrm{mg} / \mathrm{ml}$ ) durchgeführt (Technik siehe in [47,98], bildgestützt in [41]). Gleichzeitig zur Desensibilisierung bei Hyperalgesie der apikalen LSIR, wie man sie bei exazerbiertem TRS häufig sieht (Triggerpunkt: SIPS), wurde eine spezielle landmarkenorientierte fächerförmige
Infiltrationstechnik in den dorsalen Bandapparat der SIG und an den Ansätzen des Lig. iliolumbale durchgeführt (Gemisch: je Seite Triamcinolon $10 \mathrm{mg}, 5 \mathrm{ml}$ Mepivacain 0,5\%, 6-8cm Kanüle), was einer "semitargeted" fokal/regional weichteilbasierten TLI bzw. proximal periartikulären SIGInfiltration entspricht (•Tab. 5, Technik siehe in $[47,98,100])$.

\section{Therapeutische Lokal- interventionen beim TRS: Techniken, Effektivität, Evidenz}

Bei chronischem TRS ist nach Evaluation durch ein ausgedehntes Expertengremiums die Evidenz für $62 \%$ der diagnostischen und $52 \%$ der therapeutischen Interventionen moderat (angemessen) bis gut [58]. Grob orientierend lassen sich die perkutanen minimal-invasiv-interventionellen Verfahren zur mehr oder weniger gezielten Schmerztherapie beim TRS wie in - Tab. 4 dargestellt einteilen. Betrachtet man die TLI-Techniken an der LSIR im Speziellen, so besteht ein Unterschied in den therapeutischen Evidenzen.

Es ist u.a. der Verdienst der ASIPP, die anhand systematischer Literaturreviews der zurückliegenden knapp 40 Jahre (von 1966 bis 2011) hinsichtlich der Betrachtung der Effektivität im Jahr 2012 „Licht ins Dunkel“ der Evidenz bildgestützter lumbal, para- und periduraler LA-GK-Injektionen gebracht hat (- Tab. 5). Das 
Tab. 4 Übersicht etablierter interventionell-schmerztherapeutischer Techniken beim TRS

\begin{tabular}{|c|c|}
\hline & Methodik \\
\hline \multirow{8}{*}{$\begin{array}{l}\text { Fokal/regional weichteil- } \\
\text { basierte TLI } \\
\text { "semi-/untargeted“ } \\
\text { (nur } \\
\text { landmarkenorientiert }{ }^{\mathrm{a}} \text { ) }\end{array}$} & Neuraltherapeutische Injektionen/Infiltrationen: \\
\hline & Triggerpunkt-gestützte myofasziale oder \\
\hline & Leitmuskel-basierte, DAVOS-orientierte myotendinöse TLI \\
\hline & $\begin{array}{l}\text { SIPS-Region-Infiltration (nozizeptorreiche Ansatzregion der lum- } \\
\text { bosakroiliakalen Bänder), ist keine typ. SIG-Injektion }\end{array}$ \\
\hline & Prolotherapie \\
\hline & Botox-Injektionen \\
\hline & s.c.-Infiltrationen (Quaddelung) \\
\hline & Ungezielte i.m. Injektionen (meist gluteal), systemischer Ansatz \\
\hline \multirow{15}{*}{$\begin{array}{l}\text { Wirbelsäulennahe TLI } \\
\text { "targeted“ (mit oder ohne } \\
\text { Bildunterstützung }{ }^{\mathrm{a}, \mathrm{b}, \mathrm{c}, \mathrm{d})}\end{array}$} & Epidurale Injektionstechniken (EPI): \\
\hline & Kaudal-/Sakralblock ${ }^{\mathrm{a}, \mathrm{b}, \mathrm{c}, \mathrm{d}}$ \\
\hline & Transforaminal via Foramen sacrale $1^{a, b}$ (Technik des Autors) \\
\hline & Interlaminär dorsal (LOR bei Landmarkentechnik) $)^{\mathrm{a}, \mathrm{c}, \mathrm{d}}$ \\
\hline & Interlaminär rezessual-perineural| $\left.\right|^{\mathrm{a}, \mathrm{c}, \mathrm{d}, \mathrm{e}}$ \\
\hline & Extraforaminal $(=\mathrm{LSPA})^{\mathrm{a}, \mathrm{b}, \mathrm{d}, \mathrm{e}}$ \\
\hline & Transforaminal lumbal (= PRT) ${ }^{\mathrm{d}}$ \\
\hline & Facettengelenksinterventionen (FGI): \\
\hline & Perikapsulär $r^{a, b, c}$ \\
\hline & i.a./transkapsulär, d,e \\
\hline & „Medial-branch“-Block, \\
\hline & Sakroiliakalgelenk (SIG): \\
\hline & Periartikulär $r^{a, b, c}$ \\
\hline & i.a./transkapsulär $r^{a, b, c, d, e ~}$ \\
\hline & Intradiskale Injektion c,d,e \\
\hline \multirow[t]{5}{*}{$\begin{array}{l}\text { Denervationen } \\
\text { Ablationen } \\
\text { (nur bildgestützt }{ }^{\mathrm{c}, \mathrm{d}} \text { ) }\end{array}$} & $\begin{array}{l}\text { FG-Denervation/Ablation }{ }^{c, d, f} \text { : RF-Neurotomie (konventionell- } \\
\text { bipolar-cooled-gepulst). Thermokoagulation, Kryosonden, YAG- } \\
\text { Laser }\end{array}$ \\
\hline & SIG-Ablation (i.a. oder "lateral branches") c,d \\
\hline & SCS: „spinal cord stimulation“ \\
\hline & Intrathekale Opioidgabe \\
\hline & Intradiskale Ablation \\
\hline \multicolumn{2}{|c|}{$\begin{array}{l}\text { BV Bildverstärker/Bildwandler (z. B. C-Bogen), CT Cor } \\
\text { EPI epidurale Injektion, FGI Intervention am Facette } \\
\text { LOR „Ilost of resistance"-Technik (landmarkenorientie } \\
\text { nervanalgesie, PRT periradikuläre Therapie, RF Radic } \\
\text { SIPS Spina iliaca posterior superior, Sono Sonograph } \\
{ }^{a} \text { landmarkenorientiert } \\
\text { bsonographisch gestützt } \\
\text { 'Bildwandler-gestützt (z. B. C-Bogen) } \\
{ }^{\mathrm{d} C} \text { CT-gestützt (nur mit Fachkunde CT) } \\
{ }^{\mathrm{e}} \text { MRT-gestützt (klinisch noch nicht richtig etabliert) }\end{array}$} \\
\hline
\end{tabular}

geschah unter Anlage hoher Assessmentqualität und klinischer Relevanzkriterien (u.a. Cochrane-Musculoskeletal-ReviewGroup-Kriterien für randomisierte Trials bei interventionellen Techniken, NewcastleOttawa-Scale-Kriterien für fluoroskopische Beobachtungsstudien, Evidenzqualität: U.S. Preventive Services Task Force). Dabei wurden das primäre OutcomeKriterium Schmerzreduktion (in Kurzeit $<6$ Mon. und Langzeit > 6 Mon.) und se-
Evidenzevaluierung der therapeutischen Wirksamkeit wären. Wie bei der MM, besteht auch für die landmarkengestützten Injektionen hinsichtlich der Therapieeffektivität jedoch eine jahrzehntelange positive klinische Empirie bei gleichzeitig hoher Praktikabilität und Umsetzbarkeit in der Praxis am Patienten. Auch wenn diese evidenzbasierten Kriterien nicht standhalten, sind sie aus der v.a. ambulanten Versorgung des TRS nicht wegzudenken bzw. hier unentbehrlich.

Nahezu alle etablierten, zielorientierten bildgestützten wirbelsäulennahen Injektionen $[39,41,47]$ sind auch landmarkenorientiert ohne Bildunterstützung durchführbar. Voraussetzung zur Anwendung muss aber sein, dass der Anwender diese Techniken, wie bei der MM auch, beherrscht. Hier reicht es nicht aus, sich die, v.a. auf Krämer et al. [45, 90] basierenden Landmarkentechniken autodidaktisch anhand der speziellen Fachliteratur [47, 98-100] anzueignen. Es ist eine qualitätskontrollierte Schulung notwendig, um dieses Verfahren sicher und gezielt am Patienten indikationsorientiert anwenden zu können. Daher werden diesbezüglich Kurse der entsprechenden Fachgesellschaften angeboten (z.B. strukturiertes Aufbaucurriculum der IGOST). Sie sollten absolviert werden, da sie zunehmend zur Voraussetzung für die Abrechenbarkeit entsprechender Leistungen werden.

Neuraltherapeutische Infiltrationen und Injektionen beim TRS

Dies sind triggerpunktgestützte myofasziale oder leitmuskelbasierte, evtl. auch DAVOS-orientierte ("da wo's weh tut ${ }^{\prime \prime}$ ) myotendinöse TLI, die als Instrument in der ambulanten Versorgung des lokalen und pseudoradikulären Lumbalsyndroms und des SIG-Syndroms mit oder ohne Blockierung etabliertes Instrument sind. Interessanterweise gibt es auch hier keine hinreichenden Evidenzen in der Literatur, es mangelt an entsprechenden Studien guter Qualität. Typische Zielregionen der Infiltration sind die dorsolateralen Crista iliaca, SIPS-Region und paraspinös segmentale Regionen. 
Tab. 5 Effektivität und Evidenz wirbelsäulennaher bildgestützter Injektionen beim Lumbosakralsyndrom, tabellarischer Literaturreview

\begin{tabular}{|c|c|c|c|}
\hline Schmerzgenerator & Injektionstechnik 1 & Injektionstechnik 2 & Injektionstechnik 3 \\
\hline \multicolumn{4}{|c|}{ Therapeutische epidurale Injektionen (EPI) } \\
\hline Technik: & $\begin{array}{l}\text { Kaudale EPI (,kaudaler } \\
\text { Block") }\end{array}$ & Interlaminäre EPI & Transforaminale Injektion (PRT) \\
\hline $\begin{array}{l}\text { Reviews gesamt: } \\
\text { (nur bildgestützt, Ausnah- } \\
\text { me LOR) }\end{array}$ & $\begin{array}{l}\text { LA + GK: gut }[1,72], \text { überle- } \\
\text { gen [74], positiv [77] } \\
\text { nur LA: moderat [72] }\end{array}$ & $\begin{array}{l}\text { LA + GK: gut [6, 105], moderat (nur KZ) [16, } \\
\text { 77], (LOR-Technik: schlecht [71]) } \\
\text { nur LA: moderat [6] } \\
\text { nur GK: schlecht }[7,8], \text { moderat [44] }\end{array}$ & $\begin{array}{l}\text { LA+ GK: gut }[5,10,60,74], \text { moderat } \\
{[61,78], \text { Operationsvermeidung }[76,} \\
78] \\
\text { nur LA: moderat }[16,60,76] \\
\text { nur GK: schlecht }[76]\end{array}$ \\
\hline $\begin{array}{l}\text { ASIPP-Reviews, } \\
\text { 1966-2011 } \\
\text { (nur bildgestützt): }\end{array}$ & $\begin{array}{l}\text { [72] } \\
\text { inkludiert: 16, RCT: 11, } \\
\text { non- } R C T \text { (mit BV) } 5 \\
\text { revidiert: } 145 \text { von } 653\end{array}$ & $\begin{array}{l}\text { [6] } \\
\text { inkludiert: } 26, R C T:: 23 \\
\text { revidiert: } 150 \text { von } 520\end{array}$ & $\begin{array}{l}{[10,60]} \\
\text { inkludiert: } 25, R C T: 15 \\
\text { revidiert: } 117 \text { von } 428\end{array}$ \\
\hline $\begin{array}{l}\text { BSV und/oder Radikulopa- } \\
\text { thie }\end{array}$ & $\begin{array}{l}\text { LA + GK: gut LZ \& KZ } \\
\text { nur LA: moderat }\end{array}$ & $\begin{array}{l}\text { LA + GK: gut LZ \& KZ } \\
\text { nur LA: moderat }\end{array}$ & $\begin{array}{l}\text { LA + GK: gut LZ \& KZ } \\
\text { nur LA: moderat }\end{array}$ \\
\hline $\begin{array}{l}\text { Diskogener oder axialer } \\
\text { TRS }\end{array}$ & LA+GK: gut & $\begin{array}{l}\text { LA + GK: gut } \\
\text { nur LA: schlecht }\end{array}$ & $\begin{array}{l}\text { LA + GK: schlecht } \\
\text { nur LA: schlecht }\end{array}$ \\
\hline Spinalstenose & LA + GK: moderat & LA + GK: moderat & LA + GK: moderat \\
\hline "Post-surgery"-Syndrom & LA + GK: moderat & $?$ & $\begin{array}{l}\text { LA + GK: schlecht } \\
\text { nur LA: schlecht }\end{array}$ \\
\hline \multicolumn{4}{|c|}{ Therapeutische lumbale Facettengelenksinterventionen (FGI) } \\
\hline Technik: & Intraartikuläre FGI & „Medial-branch"-Block & Radiofrequenzablation \\
\hline $\begin{array}{l}\text { Reviews gesamt: } \\
\text { (nur bildgestützt, Ausnah- } \\
\text { me LOR) }\end{array}$ & $\begin{array}{l}\text { Schlecht }[15,26,62,96, \\
108]\end{array}$ & $\begin{array}{l}\text { LZ: gut [26], LZ: moderat }[9,15,23,62,96, \\
\text { 108] } \\
\text { KZ: gut }[62,108], \text { moderat [9] }\end{array}$ & $\begin{array}{l}\text { Gut [26], moderat }[15,62,96], \text { (ge- } \\
\text { pulst limitiert, } 1 \text { non-RCT) }\end{array}$ \\
\hline $\begin{array}{l}\text { ASIPP-Review, 1966-2011 } \\
\text { [26] } \\
\text { inkludiert: } 25, R C T: 11, \\
\text { non-RCT } 14 \\
\text { revidiert: } 122 \text { von } 335\end{array}$ & $\begin{array}{l}\text { Positiv: } 5 \text { non-RCT } \\
\text { negativ: } 2 \text { RCT, } 1 \text { non-RCT }\end{array}$ & $\begin{array}{l}\text { LA + GK/nur LA: positiv } \\
\text { LZ: } 1 \text { RCT, KZ: } 1 \text { RCT }\end{array}$ & $\begin{array}{l}\text { Positiv, LZ: } 6 \text { RCT LZ, } 7 \text { non-RCT } \\
\text { negativ: } 1 \text { RCT }\end{array}$ \\
\hline
\end{tabular}

\section{Epidurale Injektionen (EPI) beim TRS}

Die lumbosakrale epidurale Injektion (EPI), ist die häufigste Interventionen zur Therapie starker überregionaler TRS. Ihre Evidenz variiert je nach Techniken stark ([72]; - Tab. 5). In ihrer Subgruppenanalyse evaluierte die ASIPP nur für die Indikationsgruppe lumbaler BSV und/oder Radikulopathie eine gute Evidenz für alle 3 EPI-Verfahren (kaudaler Block, interlaminäre EPI und transforaminale EPI), wenn neben LA zusätzlich ein GK beigemischt wird; ohne GK bei nur LA-EPI besteht diesbezüglich eine moderate Evidenz. Verwendet man ein LA-GK-Gemisch, so ist die transforaminale EPI nur noch bei der Spinalstenose von moderater Evidenz; bzgl. diskogenem, axialem Schmerz und bei „Post-surgery"-Syndromen ist diese nicht empfohlen (schlechte Effektivität). Interlaminäre EPI und Kaudalblock mit LA-GK-Gemisch zeigen bei diskogenem, axialem Schmerz und Spinalstenose eine moderate Effektivität.

\section{Off-Label}

Bezüglich der EPI-Techniken muss erwähnt werden, dass bei perineuralen Injektionen die GK nicht zugelassen sind (Ausnahme: CT-gestützte PRT bei Triam-Lichtenstein). Dies steht im Gegensatz zur evidenzbasiert aufgezeigten Notwendigkeit der Zugabe von GK zum LA, um einen guten Therapieeffekt zu erreichen. Dies bedeutet, dass jegliche EPI mit GK einer OffLabel-Aufklärung und -Einwilligung durch den Patienten bedarf und nicht auf Basis der gesetzlichen Krankenversicherung abgerechnet werden darf.

\section{Strahlenschutz}

Hinsichtlich der bildgestützten Interventionen wird derzeit (noch) an der Wirbelsäule und am SIG die Verwendung strahlenintensiver Verfahren, v.a. Bildverstär- ker/-wandler (BV, oft in Form des C-Bogens) oder $\mathrm{CT}$, favorisiert. Viele Techniken sind jedoch auch sonographisch gestützt machbar und beschrieben $[56,97]$. Der MRT obliegt hier nach Auffassung des Autors in naher Zukunft, bei fehlender Strahlenbelastung und zunehmend etablierter Real-Time-Protokolle, die Rolle des Goldstandards. Bis dahin ist die Voraussetzung zur Vermeidung überhöhter Strahlenbelastung die Kenntnis der Regeln des Strahlenschutzes und der Technik des verwendeten Verfahrens. Beim BV spielt die Durchleuchtungszeit eine zentrale Rolle, was nur durch Routine bzw. gute Schulung erreicht werden kann, um die „learning curve" möglichst kurz zu halten [87, 89]. Was die CT betrifft, so ist die fachgerechte Nutzung der "Low-dose"-CT unabdingbar, um nicht nur den Behandler, sondern auch den Patienten vor einer unverhältnismäßig hohen Strahlenbelastung zu schützen. Wer also mit dosisrelevanten 

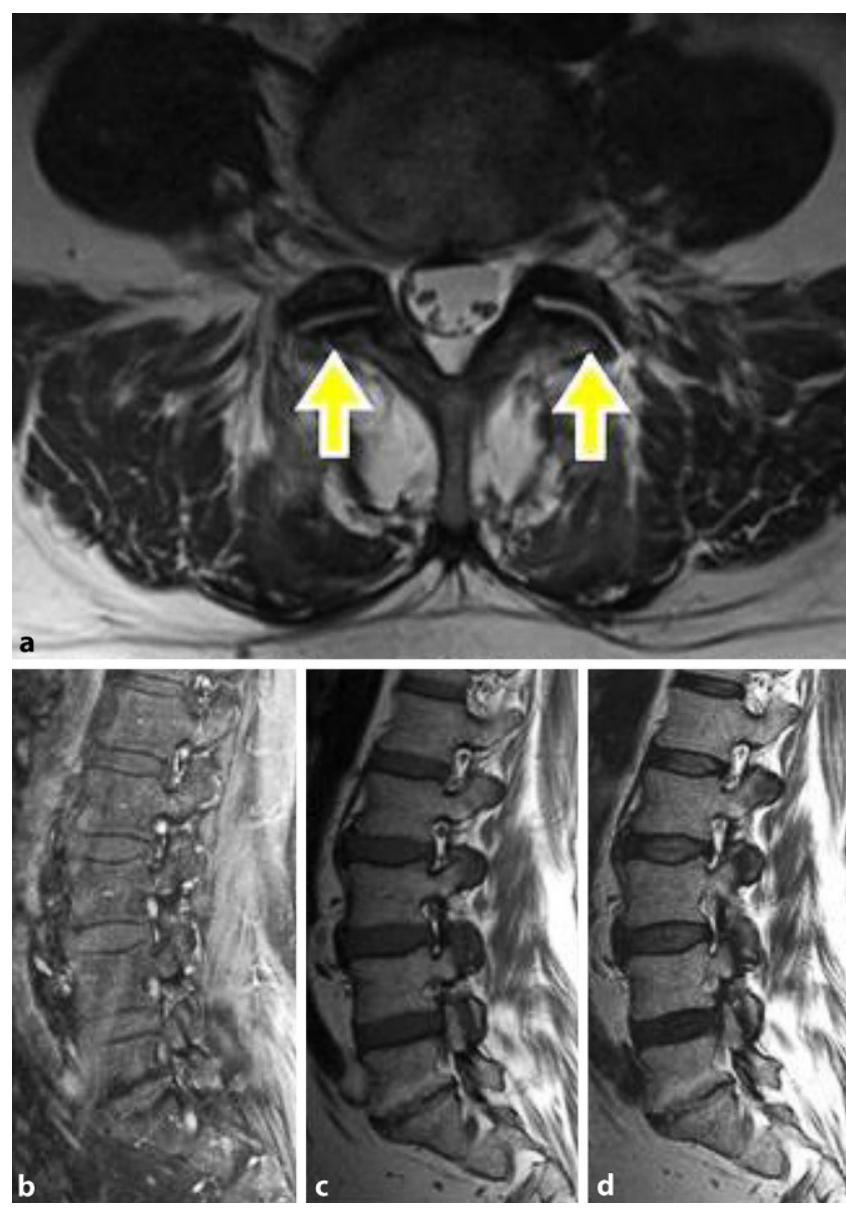

Abb. $5<$ Kasuistik - MRT der Lendenwirbelsäule. Transversal (a), Pfeile Facettengelenke $L 5 / S 1$, sagittal $\operatorname{T1w}(\mathbf{b}), \mathrm{T} 2 \mathrm{w}(\mathbf{c})$, T1w mit Fettsatturierung (d). (Mit freundl. Genehmigung, $\odot$ U. Schütz alle Rechte vorbehalten.)

Begriffen, wie "shadowing, overbeaming, overranging, pitch, windowing ", Filterkern, Rekonstruktionsalgorithmus, Schichtkollimation und SNR, nichts anfangen kann, muss die Hände von CT-gestützten Interventionen lassen, wenn kein Radiologe unterstützend zur Seite steht [83]. Nur so kann die "Low-dose"-CT-gestützte Injektion mit 0,25 mSvähnlich niedrige Strahlungsexpositionen wie die gepulste Röntgenfluoroskopie (ca. 1,0 mSv) gewährleisten [2, 14, 82, 84].

\section{Kontraindikationen}

Kontraindikationen (v.a. bei EPI-Techniken) wie "red flags", Medikamentenunverträglichkeiten, lokale trophische Störungen, lokale/systemische Infekte, Leukopenie, ZNS-Erkrankungen, schwere HerzKreislauf-Erkrankungen und Gerinnungsstörungen sind sorgfältig auszuschließen. Aufgrund möglicher (sehr seltener) schwerwiegender Komplikationen, sollte v. a. bei EPI-Techniken die direkte Notfallversorgung bis hin zum ACLS (,advanced cardiac life support") gewährleistet sein [13]. Bei Einnahme antithrombotisch wirksamer Medikamente ist die aktuelle DGAILeitlinie „Rückenmarksnahe Regionalanästhesien und Thromboembolieprophylaxe/ antithrombotische Medikation" [106] eine gute Orientierung. INR/Quick und Thrombozytenzahlen sollten Mindestwerte von

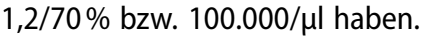

\section{Kasuistik-Teil 3: Re-Assessment nach 10 Tagen}

\section{MRT LWS, nativ}

Im Segment L5/S1 Nachweis einer fettig degenerativen Osteochondrose (Modic-Typ 2) und Spondylarthrose beidseits mit i.a. Flüssigkeit links. Fettiger Umbau der medianen tiefen paraspinalen Muskulatur, keine SIG-Signalalteration (-Abb. 5).

\section{Klinisches Bild}

Neurologie: Unauffällige Neurophysiologie für L5 und S1, Ausschluss einer Radikulopathie.
Im klinische Re-Assessment nach kaudaler EPI und Neuraltherapie SIPS beidseits deutliche Beschwerdebesserung:

- gerader Stand, keine Schmerzfehlhaltung, Hyperlordose LWS

- keine Berührungsempfindlichkeit mehr, noch deutliche Druckempfindlichkeit lumbosakral bilateral FG L5S1 bds., SIPS links, gluteal links (Valleix negativ)

- MIP: keine freie Richtung, Muskelhartspann M. lumborum bds. und gluteal links

- Lasègue negativ, Sensibilität o.B., Hyporeflexie Achillessehnenreflex/ Patellarsehnenreflex bds. unverändert symmetrisch

- Schmerzmuster: typisch für FG-Syndrom lumbosakral rechtsdominant

(• Abb. 1b)

- typischer FG-Federungsschmerz [20, 59]

Bei klinisch bildgebend hochgradigem V.a. auf Vorliegen eines aktivierten FGSyndroms L5/S1 bei chronisch degenerativem Lumbosakralsyndrom wurde der Patient über die Möglichkeit der Durchführung eines FG-Denervation/Ablation nach Diagnosesicherung über einen diagnostischen FG-Doppelblock L5/S1 informiert. Nach Aufklärung über die Funktionsbeeinträchtigung der segmentalen Mm. multifidii durch Denervation der Rr. dorsales [94], lehnte der Patient diese Maßnahmen ab und stimmte alternativ der beidseitigen GF-Infiltration L5/S1 zu, welche BV-gestützt (je FG, Gemisch: Triamcinolon 40 mg, $1 \mathrm{ml}, 10 \mathrm{ml}$ Mepivacain 0,5\%) 3-malig im Abstand von 7 Tagen leitlinienkonform durchgeführt wurde [19, 23, 34, 58].

\section{Facettengelenkinterventionen (FGI) beim TRS}

Seit Schwarzer et al. [91] gilt, dass es keine validen klinischen Kriterien bzw. keine klinischen Funktionstest gibt, welche geeignet sind, die Diagnose eines lumbalen FGS sicher zu verifizieren. Der (nichtfunktionellen) medizinischen Bildgebung (Röntgen, CT, MRT) kommt hierbei "nur" die Rolle der Ausschlussdiagnostikzu, da auch eine hierdurch detektierte starke Spondylarthrose klinisch dauerhaft symptomlos sein kann (Reliabilität und prädiktiver Wert sind ge- 
ring) [80]. Die angegebene Spannbreite hoher Prävalenzen des FGS (36-67\%) als Ursache chronifizierter TRS ist aufgrund der hohen Wahrscheinlichkeit eines positiven Testergebnisses für die Präsenz der Pathologie lumbales FG-Syndrom erklärt $[3,25]$, und man geht daher davon aus, dass dieses weniger häufig als Ursache spezifischer Kreuzschmerzen vorliegt als diagnostiziert.

Die Diagnose eines FGS als spezifischer TRS ist auf Basis der Daten der ASIPPResearch-Group ([27]: $n=325$, gesichtet 170, implementiert 23) nur dann gut valide, wenn dies durch einen Doppelblock, also eine 2-malige Durchführung eines bildgestützten FG-Blockes mit unterschiedlich lang wirksamen LA, unter einem Outcome-Parameter von mind. $75 \%$ Schmerzreduktion eruiert wird. Doppelblocks mit Outcome 50-74\% Schmerzreduktion sind, ebenso wie Kontrollblocks mit Placebo, in ihrer diagnostischen Aussagekraft moderat bis schlecht [88], also nicht indiziert. Einzelblocks sind als diagnostisches Verfahren obsolet, da schlecht in ihrer Evidenz [27].

Betrachtet man die therapeutischen FGI, so sind intraartikuläre FG-Injektionen, egal ob mit oder ohne GK, in ihrer Effektivität hinsichtlich der Schmerzreduktion als schlecht zu bewerten, wohingegen der „Medial-branch"-Block bei serieller Durchführung (3- bis 6-malig) bzgl. der Kurzund Langzeitwirkung gute Evidenzen aufzeigt. Gleiches gilt für die Radiofrequenzneurotomie (RFN) des lumbalen FG (- Tab. 5), wobei die gepulste RFN hier nur limitierte Ergebnisse aufzeigt. Diese Daten beziehen sich durchweg auf bild-, meist röntgengestützte Interventionen, bzgl. Sonographie-, CT- und landmarkengestützte Verfahren liegen keine suffizienten Studiendaten oder Evidenzangaben vor.

Interventionen am Sakroiliakalgelenk beim TRS

Die Rolle des SIG-Syndroms beim TRS ist intensiv erforscht. Die Innervation des SIG ist komplex und, wie seine Größe und Form (Heterogenität), individuell sehr variabel [18]. Dadurch kann das SIG als Schmerzgenerator im klinischen Bild (SIG-Syndrom), wie ein Chamäleon, durch unterschied- lichste Schmerzmuster in der LSIR bis in die Oberschenkel und die Leisten reichend imponieren [38]. Daher kann, ähnlich wie beim FGS, weder die Anamnese noch die klinisch-funktionelle Untersuchung und/ oder ein radiologisches Merkmal die Diagnose SIG-verursachter Schmerz sicherstellen. Dies erklärt, warum die Prävalenz von SIG-Schmerzen je Studiendesign breit zwischen 10 und $62 \%$ streut. Die Mehrheit der von Simopoulos et al. [93] analysierten Studien von 1966-2012 ( $n=2736$, gesichtet 450, 18 implementiert), deutet auf eine Punktprävalenz von ca. $25 \%$ hin.

Eine diagnostische SIG-Blockade kann nur durch sichere i.a.-Injektion ins SIG durchgeführt werden, was nur bildgestützt (BV, CT, MRT) möglich ist. Bildgestützt durchgeführte unkontrollierte SIGBlocks zeigen eine falsch-positive Rate von ca. $20 \%$. Hingegen ist, wie auch beim FGS, die Evidenz für den kontrollierte SIGDoppelblock (vergleichende LA-Blockaden bei Outcome-Kriterium mindestens $70 \%$ Schmerzreduktion) im systematischen Review von Simopoulos et al. [93] gut, die für provokative diagnostische Tests nur mäßig.

Therapeutisch werden intra- und periartikuläre Injektionen, Sakralastblöcke und HF-Ablation, sowohl landmarken- als auch bildgestützt, verwendet, um Patienten mit SIG-Schmerzen zu helfen ([95], - Tab. 4). Diese interventionsbasierte Therapie des SIG-Syndroms ist durch eine große Variabilität und einen Mangel an qualitativ hochwertigen Studien gekennzeichnet, sodass hier keine validen Ergebnisse eruierbar sind. Die vorliegenden klinischen Studien beschreiben einen mittelfristigen Nutzen sowohl für intra- als auch extraartikuläre GK-Injektionen [38]. Bei Patienten, die keine anhaltende Linderung durch SIGInjektionen erfahren, kann die RF-Denervation eventuell eine Linderung von bis zu 1 Jahr bewirken.

\section{Sonstige interventionelle Verfahren beim TRS}

Prolotherapie, Botulinumtoxin (Btx) Auf die Verfahren der Prolotherapie (hyperosmolare Dextrose) und der Btx-Injektionen kann hier hinsichtlich Durchführung, Wirkungsweise, Effekt und Evidenz nicht näher eingegangen werden. Für diese The- rapieformen besteht für den TRS keine Evidenz, da es an entsprechenden Studien mangelt bzw. die Fallzahlen zu gering sind [95]. Im Bereich der lumbalen FG und der paravertebralen Muskulatur werden sie als eher ineffektiv beschrieben [17, 33]. Metaanalysen sind bis dato nicht möglich [104]. Einzelstudien bzgl. dem SIG sind vielversprechend: Eine prospektive Fall-KontrollStudie von Lee und Kollegen [49], welche die BV-gestützte periartikuläre Btx-SIG-Injektion mit der KG-LA-Injektion verglich, kam in der die klinische Wirksamkeit zu dem Ergebnis, dass bei der Schmerzreduktion im 1. postinterventionellen Monat kein Unterschied bestand, jedoch in der Btx-Gruppe in der Nachbeobachtung nach 2 und 3 Monaten im Vergleich signifikant anhielt. Auf die entsprechende Fachliteratur wird verwiesen $[49,50,95]$.

\section{Intradiskale Interventionen (IDT)}

Die intradiskalen Interventionen (IDT) mit GK und PRP („platteled enriched plasma“) sind in den Hintergrund geraten, da die nur kurzfristigen positiven schmerzhemmenden Effekte den vergleichsweise hohen Prozedurenaufwand im Ergebnis nicht rechtfertigen $[22,64]$. Die intradiskalen Ablationen bewirken oftmals aufgrund der iatrogenen Gewebeschädigung einen Progress der Diskopathie mit all deren das gesamte Segment betreffenden möglichen Folgen (s. oben). Die IDT mit Ozon $\left(\mathrm{O}_{2} / \mathrm{O}_{3}\right)$ scheint bei monosegmentaler Diskopathie vergleichsweise positive Effekte bzw. Empfehlungsgrade zu haben $[35,57,69]$.

\section{Kasuistik-Teil 4: Re-Assessment, 4. Woche}

\section{Klinisches Bild}

Subjektiv noch diskreter residueller bewegungsabhängiger Schmerz lumbosakral links:

- diskrete Druckempfindlichkeit lumbosakral bilateral über FG L5/S1 links (kein FG-Federungsschmerz)

- MIP: freie Richtung (L4+ li, lo), Muskelhartspann M. lumborum links

\section{Procedere}

Manipulation (L4 re, ky):

- Krankengymnastik: entlordosierendstabilisierend zur Anleitung (,Rückenschule") 


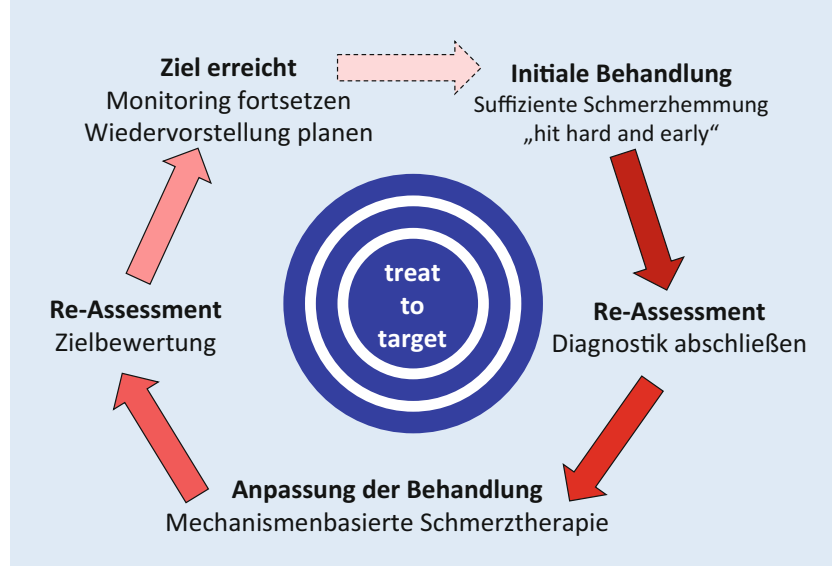

- Ergotherapie: Ergonomie am Arbeitsplatz

- Akupunktur: Regulation inhibitorischer Systeme, Desensibilisierung

Weiter Verlauf in 2 Jahren

- noch 2-malig funktionelle Lumbosakralgie auf niedrigem Niveau

- "back to work“ 6 Wochen nach Erstvorstellung, keine weitere Arbeitsunfähigkeit: innerbetrieblicher Bereichswechsel (keine regelhaften Transferarbeiten mehr)

- Einleitung hausärztlich supervidierte Gewichtsreduktion

- Tennis moderat

\section{Zusammenfassung}

Ein primäres funktionelles lumbosakrales Schmerzmuster beim degenerativen TRS ist, wenn keine Kontraindikationen vorliegen, eine Indikation für eine initiale direkte MM-Behandlung, da in diesem Falle die Blockierung und/oder die myofasziale Dysfunktion die Ursache spezifischer TRS ist, da primärer Schmerzgenerator. Ist das funktionelle Schmerzmuster jedoch Folge strukturell-degenerativer Prozesse, so ist das funktionelle Schmerzmuster unspezifisch, da sich der primäre Schmerzgenerator auf strukturpathologischer Ebene befindet und somit Ursache spezifischer TRS ist (z. B. degeneratives FGS, erosive Osteochondrose). Diese Differenzierung wird in der Leitlinie "Spezifischer Kreuzschmerz" [11] jedoch nicht getroffen, was in der Weiterentwicklung nachzuholen sein wird. Insofern kann man die Kritiker der in derzeitiger Form vorliegenden
Abb. $6 \triangleleft$ Zielorientierte ambulante Schmerztherapie bei chronischem, degenerativem tiefem Rückenschmerz. (Mit freundl. Genehmigung, ( ) U. Schütz, alle Rechte vorbehalten.)

S2K-Leitlinie verstehen, wenn sie die funktionellen Schmerzmuster bei der Diagnose "Nichtspezifischer Kreuzschmerz" [52] weiter angesiedelt sehen (wollen).

Angelehnt an die zielorientierte Therapieplanung und -führung in der Versorgung chronisch Rheumakranker [12, 75], plädiert der Autor bei Patienten mit (chronischem) degenerativem TRS orientiert an den differenzialtherapeutischen Grundsätzen der MM [55] unter Kenntnis von Techniken und Evidenzen der TLI (• Tab. 5), für die Etablierung eines strukturierten mechanismenbasierten (- Abb. 5; [52]) Therapiekonzeptes im Sinne des "treat to target ${ }^{\prime}$ (T2T) im ambulanten schmerztherapeutischen Versorgungsbereich (• Abb. 6).

\section{》) Der Autor plädiert für die Etablierung eines strukturierten mechanismenbasierten Therapiekonzeptes}

Dies setzt eine konsequente (Primär-)Diagnostik mit Schmerzanalyse unter der Prämisse, dass TRS, wenn strukturell-funktionell bedingt, immer spezifisch ist [2], voraus. Das heißt, die breite Anamnese (auch soziofamiliär zur Detektion von "yellow, red, blue, black flags"), mit strukturbezogener (Ausschluss-)Diagnostik (klinisch-bildgebend) und funktioneller Differenzierung (nach MM-Grundsätzen, ggf. interventionelle Blocks), sollte am Ende zur Formulierung einer 3-Ebenen-Diagnose als Voraussetzung zur mechanismenbasiert-zielorientierten hierarchischen Stufentherapie führen:
1. Initial wirksame symptomatische Schmerzbekämpfung (,hit hard and early"):

- z.B. breite Neuraltherapie, peridurale $\mathrm{TLI}$, analgetisch-antiphlogistische Infusion

2. idealerweise Detektierung und Stilllegung/Wegnahme des primären Nozigenerators (initiales T2T):

- z. B. gezielte, ggf. bildgestützte TLI (MM, wenn rein funktionelles Schmerzmuster)

3. Beseitigung residueller, reaktiver (sekundärer) funktioneller Schmerzmuster (myofasziale und/oder hypomobile Dysfunktion der LSIR):

- z.B. MM: Mobilisation, Manipulation, TLI: Neuraltherapie

4. bei (beginnender) Chronifizierung: mechanismenbasierte multimodale Schmerztherapie (prolongiertes T2T):

- Rückbau von Chronifizierungsvorgängen (Desensibilisierung): z. B. serielle TLI, medikamentöse Analgesie, ggf. Opiate (LONTS [36])

- Rekonditionierung der inhibitorischen Systeme: z. B. Akupunktur, Verhaltenstherapie

5. Muskuläre Rekonditionierung (Funktion, Balance) und Rekoordination der motorischen Systeme:

- z.B. aktive Krankengymnastik, Ergotherapie, Krankengymnastik am Gerät, ggf. ambulante/stationäre Rehabilitation

6. Regelhaftes Training der zurückoder neugewonnenen Funktion als Rezidivprophylaxe (liegt in der Eigenverantwortung des Patienten)

\section{Schlussfolgerungen}

\section{TRS}

- ist meistens spezifisch, d. h. er hat eine strukturelle oder funktionelle primäre Ursache. Nur selten ist er „nichtspezifisch"; oft mangelt es nur an der diagnostischen Sorgfalt und Konsequenz.

- bedarf einer zielgerichteten, ursachenspezifischen Therapie („treat to target ${ }^{\prime \prime}$ ).

- bedarf initial einer raschen schmerzhemmenden Intervention („hit hard an early"). 
- ist im postakuten Stadium selten mono-, sondern meistens multikausal (komplex, mehrere Schmerzgeneratoren): myofasziale/segmentale Dysfunktion ist oft sekundär, also Folge unterschiedlicher primärer Schmerzgeneratoren und dann bereits Ausdruck beginnender Chronifizierung.

- bedarf einer differenzierten Schmerzanalyse und mehrschichtiger Diagnosenstellung (3 Ebenen).

- setzt zur Behandlung vertiefte Kenntnisse in der Schmerzphysiologie voraus.

- bedarf bei Chronifizierung eines multimodalen Behandlungskonzeptes („Leader": Spezieller Schmerztherapeut).

\section{TLI}

- relevantes diagnostisches Tool zur Spezifizierung von TRS

- relevantes initial therapeutisches Tool bei akuten, exazerbierten TRS („hits hard and early")

- relevantes Tool für gezielte therapeutische Intervention am primären Schmerzgenerator ("treats to target ${ }^{\prime \prime}$ )

- möglicher "gate opener" für MM bei sekundärem funktionellem TRS

- relevante Methode zur Down-Regulierung chronisch-struktureller und -funktioneller TRS auf niedriges Niveau (Desensibilisierung: serielle Injektionen)

- hat bei qualifizierter Anwendung weniger unerwünschte Wirkungen im Vergleich zu dauerhafter medikamentöser Analgesie

- wichtige Alternative bei Ausschöpfung medikamentöser Analgesie (Unverträglichkeiten, Risikofaktoren)

Schlussfolgernd kann konstatiert werden, dass bei sinnvollem Einsatz von Techniken der TLI und MM im Sinne eines bi-/ multimodalen Settings der degenerative (chronische) TRS rascher, gezielter und damit effektiver therapiert werden kann, auch wenn bzgl. der Therapieeffektivität bis dato v.a. die Mobilisationsmethoden der MM (und auch der Osteopathie), als auch die weichteil-neuraltherapeutischen, wie auch die landmarken-, sonographieund CT-gestützten TLI einen schlechten bzw. fehlenden Evidenznachweis haben.
Manipulative MM-Verfahren sind bis dato als mäßig effektiv evidenzbasiert bewertet. Lediglich bei röntgengestützten EPI-, FGI- und SIG-Infiltrationen sind unter Verwendung von LA-GK-Gemischen gute (bis moderate) Wirknachweise vorhanden. Gründe für die schlechten Evidenzlagen scheint es mehrere zu geben. Der Mangel an guten und das Vorhandensein von schlechte Studienqualitäten mit fehlender Bildung an RCT liegt einerseits daran, dass v.a. in der MM die Bildung von "Sham"-/Placebogruppen methodenbedingt schwierig ist. Anderseits sind bisherige (Sub-)Gruppierungen gescheitert oder mangelhaft aufgrund einer unpräzisen Diagnostik (Mischdiagnosen) bei variabler Symptomatik bzw. Multifaktorialität möglicher Schmerzursachen und uneinheitlicher Terminologie, aber auch aufgrund indifferenter Therapiesettings und variabler Therapeutenqualität.

Es besteht die Notwendigkeit der Etablierung spezifischer subgruppenbasierter RCT und Metaanalysen. Voraussetzung zu Bildung solcher Gruppen wäre jedoch eine präzise, konsequente standardisierte Diagnostik auf Basis einer weiterzuführenden einheitlichen Terminologie, wenngleich diesbezüglich in den letzten Jahren durch das Engagement entsprechender Fachorganisationen (IGOST, DGMM, SAMM, DAAO) schon viel erreicht wurde.

\section{Fazit für die Praxis}

- Ohne vertiefte Kenntnis (patho-)physiologischer neuronaler Schmerzentstehungsund -verarbeitungsmechanismen ist eine gezielte und effektive Behandlung (sub-)chronischer tiefer Rückenschmerzen (TRS) nicht möglich.

- Werden konservative/interventionelle Methoden, wie Manuelle Medizin (MM) und therapeutische Lokalinfiltration (TLI), lediglich symptomatisch orientiert bezüglich sekundärer (funktioneller) Schmerzgeneratoren eingesetzt, besteht die Gefahr der (iatrogenen) Schmerzchronifizierung; dies meist aufgrund insuffizienter Primär- oder Folgediagnostik (Re-Assessment).

- Die Detektion der primären Schmerzgeneratoren ist Voraussetzung für eine erfolgreiche mechanismenbasierte Therapie beim spezifischen TRS.

- MM und TLI sind empiriebasierte und partiell evidenzbasierte, wirkungsvolle Instrumente in der Behandlung akuter und chronischer spezifischer TRS, wel- che bei mechanismenbasiert stadiengerechtem, gezielt synergistischem bi-/ multimodalem Einsatz eine effektive Therapie und Chronifizierungsprophylaxe gewährleisten können.

- Monomodale Therapiekonzepte werden dem postakuten, rezidivierenden TRS selten gerecht und bergen eine hohe Gefahr der Chronifizierung.

\section{Korrespondenzadresse}

Prof. Dr. Uwe H. W. Schütz

Schmerztherapie und Rheumatologie, Orthopädie am Grünen Turm

Grüner-Turm-Str. 4-10, 88212 Ravensburg,

Deutschland

uwe.schuetz@orthopaedie-rv.de

\section{Einhaltung ethischer Richtlinien}

Interessenkonflikt. U.H.W. Schütz gibt an, dass kein Interessenkonflikt besteht.

Für diesen Beitrag wurden von den Autoren keine Studien an Menschen oder Tieren durchgeführt. Für die aufgeführten Studien gelten die jeweils dort angegebenen ethischen Richtlinien.

Open Access. Dieser Artikel wird unter der Creative Commons Namensnennung 4.0 International Lizenz veröffentlicht, welche die Nutzung, Vervielfältigung, Bearbeitung, Verbreitung und Wiedergabe in jeglichem Medium und Format erlaubt, sofern Sie den/die ursprünglichen Autor(en) und die Quelle ordnungsgemäß nennen, einen Link zur Creative Commons Lizenz beifügen und angeben, ob Änderungen vorgenommen wurden.

Die in diesem Artikel enthaltenen Bilder und sonstiges Drittmaterial unterliegen ebenfalls der genannten Creative Commons Lizenz, sofern sich aus der Abbildungslegende nichts anderes ergibt. Sofern das betreffende Material nicht unter der genannten Creative Commons Lizenz steht und die betreffende Handlung nicht nach gesetzlichen Vorschriften erlaubt ist, ist für die oben aufgeführten Weiterverwendungen des Materials die Einwilligung des jeweiligen Rechteinhabers einzuholen.

Weitere Details zur Lizenz entnehmen Sie bitte der Lizenzinformation auf http://creativecommons.org/ licenses/by/4.0/deed.de.

\section{Literatur}

1. American Society of Anesthesiologists Task Force on Chronic Pain Management, American Society of Regional Anesthesia and Pain Medicine (2010) Practice guidelines for chronic pain management: an updated report by the American Society of Anesthesiologists Task Force on Chronic Pain Management and the American Society of Regional Anesthesia and Pain Medicine. Anesthesiology 112(4):810-833. https://doi.org/ 10.1097/ALN.0b013e3181c43103

2. Artner J, Lattig F, Reichel H, Cakir B (2012) Effective dose of CT-guided epidural and periradicular 
injections of the lumbar spine: a retrospective study. Open Orthop J 6:357-361. https://doi.org/ 10.2174/1874325001206010357

3. Atluri S, Datta S, Falco FJ, Lee M (2008) Systematic review of diagnostic utility and therapeutic effectiveness of thoracic facet joint interventions. Pain Phys 11(5):611-629 (Review)

4. Baron R, Binder A (2004) Wie neuropathisch ist die Lumboischialgie? Das Mixed-pain-Konzept. [How neuropathic is sciatica? The mixed pain concept]. Orthopade 33:568-575. https://doi.org/10.1007/ s00132-004-0645-0

5. Benny B, Azari P (2011) The efficacy of lumbosacral transforaminal epidural steroid injections: a comprehensive literature review. J Back Musculoskelet Rehabil 24(2):67-76. https://doi.org/10.3233/ BMR-2011-0279

6. Benyamin RM, Manchikanti L, Parr AT, Diwan S, Singh V, Falco FJ, Datta S, Abdi S, Hirsch JA (2012) The effectiveness of lumbar interlaminar epidural injections in managing chronic low back and lower extremity pain. Pain Phys 15(4):E363-E404 (Review)

7. Bogduk N (1999) Epidural steroids for low back pain and sciatica. Pain Dig 9:226-227

8. Bogduk N, Christophidis N, Cherry D (1994) Epidural use of steroids in the management of back pain. Report of working party on epidural use of steroids in the management of back pain. Canberra National Health and Medical Research Council, Commonwealth of Australia, S1-76

9. Boswell MV, Colson JD, Sehgal N, Dunbar EE, Epter R (2007) A systematic review of therapeutic facet joint interventions in chronic spinal pain. Pain Phys 10(1):229-253 (Review)

10. Buenaventura RM, Datta $S$, Abdi $S$, Smith HS (2009) Systematic review of therapeutic lumbar transforaminal epidural steroid injections. Pain Phys 12(1):233-251 (Review)

11. Bundesärztekammer, Kassenärztliche Bundesvereinigung, Arbeitsgemeinschaft der wissenschaftlichen medizinischen Fachgesellschaften (2017) Nationale Versorgungsleitlinie nichtspezifischer Kreuzschmerz (NVL-NsKS), Langfassung, 2. Aufl. Version1, AWMF-Register-Nr.: nvl-007. http://www.leitlinien.de/themen/kreuzschmerz/ 2-auflage.Zugegriffen: 1.Jan. 2022

12. Burmester GR, Pope JE (2017) Novel treatment strategies in rheumatoid arthritis. Lancet 389(10086):2338-2348. https://doi.org/10.1016/ S0140-6736(17)31491-5

13. Cassuto J, Sinclair R, Bonderovic M (2006) Antiinflammatory properties of local anesthetics and their present and potential clinical implications. Acta Anesthesiol Scand 50:265-282. https://doi. org/10.1111/j.1399-6576.2006.00936.X

14. Chang AL, SchoenfeldAH, BrookAL,MillerTS (2013) Radiation dose for 345 CT-guided interlaminar lumbar epidural steroid injections. AJNR Am J Neuroradiol 34(10):1882-1886. https://doi.org/ 10.3174/ajnr.A3540

15. Chou R, Atlas SJ, Loeser JD, Rosenquist RW, Stanos SP (2011) Guideline warfare over interventional therapies for low back pain: can we raise the level of discourse? J Pain 12(8):833-839. https:// doi.org/10.1016/j.jpain.2011.04.012

16. Chou H (2009) Guideline for the evaluation and management of LBP: evidence review. American Pain Society, Glenview (Review)

17. Cogné $M$, Petit $H$, Creuzé $A$, Liguoro $D$, de Seze M (2017) Are paraspinous intramuscular injections of botulinum toxin a (BoNT-A) efficient in the treatment of chronic low-back pain? A randomised, double-blinded crossover trial. BMC
Musculoskelet Disord 18(1):454. https://doi.org/ 10.1186/s12891-017-1816-6

18. Cohen SP, Chen Y, Neufeld NJ (2013) Sacroiliac joint pain: a comprehensive review of epidemiology, diagnosis and treatment. Expert Rev Neurother 13(1):99-116. https://doi.org/10.1586/ern.12.148

19. Cohen SP, Huang JH, Brummett C (2013) Facet joint pain-advances in patient selection and treatment. Nat Rev Rheumatol 9(2):101-116. https:// doi.org/10.1038/nrrheum.2012.198 (Review)

20. Cohen SP, Raja SN (2007) Pathogenesis, diagnosis, and treatment of lumbar zygapophysial (facet) joint pain. Anesthesiology 106(3):591-614. https://doi.org/10.1097/00000542-20070300000024 (Review)

21. Cunha C, Silva AJ, Pereira P, Vaz R, Gonçalves RM, Barbosa MA (2018) The inflammatory response in the regression of lumbar disc herniation. Arthritis Res Ther 20(1):251. https://doi.org/10. 1186/s13075-018-1743-4 (Review)

22. Daste C, Laclau S, Boisson M, Segretin F, Feydy A, Lefèvre-Colau MM, Rannou F, Nguyen C (2021) Intervertebral disc therapies for non-specific chronic low back pain: a systematic review and meta-analysis. Ther Adv Musculoskelet Dis 13:1759720X211028001.https://doi.org/10.1177/ 1759720X211028001 (Review)

23. Datta S, Lee M, Falco FJ, Bryce DA, Hayek SM (2009) Systematic assessment of diagnostic accuracy and therapeutic utility of lumbar facet joint interventions. Pain Phys 12(2):437-460 (Review)

24. Donnelly JM, Hortman-Camilo $D$, Thomas $P$ (2011) Quadratum Lumborum muscle and gluteus medius muscle. In: Donely JM (Hrsg) Travell IG, Simons \& Simons' myofascial pain and dysfunction: the trigger point manual, 3. Aufl. Lippincott, , $\mathrm{S}$ 1162-1192, 1306-1328

25. Falco FJ, Datta S, Manchikanti L, Sehgal N, GeffertS, Singh V, Smith HS, Boswell MV (2012) An updated review of the diagnostic utility of cervical facet joint injections. Pain Phys 15(6):E807-E838

26. Falco FJ, Manchikanti L, DattaS, Sehgal N, GeffertS, Onyewu O, Zhu J, Coubarous S, Hameed M, Ward SP, Sharma M, Hameed H, Singh V, Boswell MV (2012) An update of the effectiveness of therapeutic lumbar facet joint interventions. Pain Phys 15(6):E909-E953 (Review)

27. Falco FJ, Manchikanti L, Datta S, Sehgal N, GeffertS, Onyewu $O$, Singh V, Bryce DA, Benyamin RM, Simopoulos TT, Vallejo R, Gupta S, Ward SP, Hirsch JA (2012) An update of the systematic assessment of the diagnostic accuracy of lumbar facetjointnerveblocks. Pain Phys 15(6):E869-E907 (Review)

28. Finnegan M, Gebhardt MM, Freeman JL (2011) Thoracolumbar paraspinal muscles. In: Donely JM (Hrsg) Travell IG, Simons \& Simons' myofascial pain and dysfunction: the trigger point manual, 3 . Aufl. Lippincott, , S1099-1126

29. Franke H, Franke JD, Fryer G (2014) Osteopathic manipulative treatment for nonspecific low back pain: a systematic review and meta-analysis. BMC Musculoskelet Disord 15:286. https://doi.org/10. 1186/1471-2474-15-286

30. Franke H, Fryer G, Ostelo RW, Kamper SJ (2015) Muscle energy technique for non-specific lowback pain. Cochrane Database Syst Rev. https:// doi.org/10.1002/14651858.CD009852.pub2

31. Freynhagen R, Baron R, Gockel U, Tölle TR (2006) painDETECT: a new screening questionnaire to identify neuropathic components in patients with back pain. Curr Med Res Opin 22(10):1911-1920. https://doi.org/10.1185/030079906X132488
32. Gerbershagen HU (1996) Das Mainzer Stadienkonzept des Schmerzes: Eine Standortbestimmung. In: Klingler D, Morawetz R, Thoden U et al (Hrsg) Antidepressiva als Analgetika. Aarachne, Wien, S71-95

33. Giordano L, Murrell WD, Maffulli N (2021) Prolotherapy for chronic low back pain: a review of literature. Br Med Bull 138(1):96-111. https://doi. org/10.1093/bmb/ldab004 (Review)

34. Halder A, Kroppenstedt S, Locher Hetal (2018) S2kLeitlinie Spezifischer Kreuzschmerz. In: Deutsche Gesellschaft für Orthopädie und Orthopädische Chirurgie (Hrsg) Leitlinien für Diagnostik und Therapie in der Orthopädie. AWMF Register-Nr.: 033-051, Bd. 06 (http://www.awmf.org/leitlinien/ detail/l//033-051.html. Zugegriffen 01. Januar 2022)

35. Hashemi M, Poorfarokh M, Mohajerani SA, Jalili P, Akhyani V, Barikani A, Farivar F (2014) Injection of intradiscal $02-03$ to reduce pain and disability of patients with low back pain due to prolapsed lumbar disk. Anesth Pain Med 4(5):e19206. https:// doi.org/10.5812/aapm.19206

36. Häuser W, Bock $F$, Hüppe $M$, Nothacker $M$, Norda H, Radbruch L, Schiltenwolf M, Schuler M, Tölle T, Viniol A, Petzke F (2020) Empfehlungen der zweiten Aktualisierung der Leitlinie LONTS. Langzeitanwendung von Opioiden bei chronischen nicht-tumorbedingten Schmerzen. Schmerz 34(3):204-244. https://doi.org/10.1007/ s00482-020-00472-y

37. Jerosch J (2007) Klinische Befunderhebung. In: Heisel J, Jerosch J (Hrsg) Schmerztherapie der Halte- und Bewegungsorgane. Allgemeine und spezielle Schmerztherapie. Springer, Heidelberg, S 28-30. ISBN 978-3-540-29890-8

38. Jung JH, Kim HI, Shin DA, Shin DG, Lee JO, Kim HJ, Chung JH (2007) Usefulness of pain distribution pattern assessment in decision-making for the patients with lumbar zygapophyseal and sacroiliac joint arthropathy. J Korean Med Sci 22:1048-1054

39. Khan SM, van der Heuvel SAS, del Rey MLR, Fenech CA, Hernandez Porras CB (2020) Lumbar procedures. In: Stogicza AR, Mansano AM, Trescot AM, Staats PS (Hrsg) Interventional pain A step-by-step guide for the FIPP exam. Springer, Berlin Heidelberg, S 109-153. ISBN 978-3-03031741-6

40. Kiter E, Karaboyun T, Tufan AC, Acar K (2010) Immunohistochemical demonstration of nerve endings in iliolumbar ligament. Spine 35(4):E101-E104. https://doi.org/10.1097/BRS.0b013e3181ae561d

41. Klessinger S, Legat $M$ (2020) Interventionen an der LWS. In: Klessinger S, Legat M, Schneider M (Hrsg) InterventionelleSchmerztherapieder Wirbelsäule. DeGruyter, , S87-122. ISBN 978-3-11-055908-8

42. Knoop J, Rutten G, Lever C, Leemeijer J, de Jong LJ, Verhagen AP, van Lankveld W, Staal JB (2021) Lack of consensus across clinical guidelines regarding the role of psychosocial factors within low back pain care: a systematic review. J Pain 22(12):1545-1559. https://doi.org/10.1016/j. jpain.2021.04.013

43. Kochen MM, Blozik E, Scherer M, Chenot JF (2009) Imaging for low-back pain. Lancet 373(9662):436-437. https://doi.org/10.1016/ S0140-6736(09)60149-5

44. Koes BW, Scholten RJ, Mens JMA, Bouter LM (1995) Efficacy of epidural steroid injections for low-back pain and sciatica: a systematic review of randomized clinical trials. Pain 63(3):279-288. https://doi.org/10.1016/0304-3959(95)00124-7

45. Krämer J (1996) Orthopädische Schmerztherapie. Dtsch Arztebl 93:1961-1965 
46. Krämer J (2002) Behandlung lumbalerWurzelkompressionssyndrome. Dtsch Arztebl 99:1510-1516

47. Lange $A$ (2012) Injektionsverfahren im Bereich der Lendenwirbelsäule. OUP 4:224-237. https://doi. org/10.3238/oup.2012.0484-0494

48. Lange A, Eckardt A (2011) Injektionsverfahren an der Lendenwirbelsäule. In: Eckardt A (Hrsg) Praxis LWS-Erkrankungen. Diagnose und Therapie. Springer, Berlin, S 186-242. ISBN 978-3-54088507-8

49. Lee JH,LeeSH,Song SH(2010)Clinical effectiveness of botulinum toxin A compared to a mixture of steroid and local anesthetics as a treatment for sacroiliac joint pain. Pain Med 11(5):692-700. https://doi.org/10.1111/j.1526-4637.2010.00838. $\mathrm{x}$

50. Gemäß Beschluss des Vorstandes der Bundesärztekammer (2007) Leitlinien der Bundesärztekammer zur Qualitätssicherung in der Röntgendiagnostik. Qualitätskriterien röntgendiagnostischer Untersuchungen. http:// www.bundesaerztekammer.de/fileadmin/user upload/downloads/LeitRoentgen2008Korr2.pdf. Zugegriffen: 10. Jan. 2022

51. Locher H (2011) Manuelle Medizin/Chriotherapie. In: Locher H, Casser HR, Strohmeier M, Grifka J (Hrsg) Spezielle Schmerztherapie der Halte- und Bewegungsorgane. Thieme, Stuttgart, New York, S 151-156

52. Locher H (2021) Manuelle Medizin, manuelle Therapie: Grundlagen, Wirkmechanismen, Indikationen und Evidenz. Unfallchirurg 124(6):433-445 https://doi.org/10.1007/s00113-021-01004-8

53. Locher H, Moll H (2017) Manuelle Medizin, Chirotherapie, Osteopathische Verfahren. In: Psczolla M Kladny B, Flechtenmacher J, Hoffmann R, Dreinhöfer D (Hrsg) Weißbuch Konservative Orthopädie und Unfallchirurgie. DeGruyter, , S 160-163. ISBN 978-3-11-053433-7

54. Locher H, Strohmeier M, Wolber K (2007) Orthopädische Schmerztherapie. In: Bischoff HP, Heisel J, Locher $\mathrm{H}$ (Hrsg) Praxis der konservativen Orthopädie. Thieme, Stuttgart, S181-198

55. Locher M, Böhni U (2015) Ableitung einer optimierten rationale Differenzialtherapie. In: Böhni U, Lauper M, Locher H (Hrsg) Manuelle Medizin 1, S 324-325 https://doi.org/10.1055/b0034-97507

56. Loizides A, Peer S, Plaikner M, Spiss V, Galiano K, Obernauer J, Gruber H (2011) Ultrasound-guided injections in the lumbar spine. Med Ultrason 13(1):54-58 (Erratum in: Med Ultrason. 2011 Jun;13(2):178)

57. Magalhaes FN, Dotta L, Sasse A, Teixera MJ, Fonoff ET (2012) Ozone therapy as a treatment for low back pain secondary to herniated disc: a systematic review and meta-analysis of randomized controlled trials. Pain Phys 15(2):E115-E129 (Review)

58. Manchikanti L, Abdi S, Atluri S, Benyamin RM, Boswell MV, Buenaventura RM, Bryce DA, Burks PA, Caraway DL, Calodney AK, Cash KA, Christo PJ, Cohen SP, Colson J, Conn A, CordnerH, CoubarousS, Datta S, Deer TR, Diwan S, Falco FJ, Fellows B, GeffertS, Grider JS, GuptaS, Hameed H, Hameed M, Hansen $H$, Helm S 2nd, Janata JW, Justiz R, Kaye AD, Lee M, Manchikanti KN, McManus CD, Onyewu O, Parr AT, Patel VB, Racz GB, Sehgal N, Sharma ML, Simopoulos TT, Singh V, Smith HS, Snook LT, Swicegood JR, Vallejo R, Ward SP, Wargo BW, Zhu J, Hirsch JA (2013) An update of comprehensive evidence-based guidelines for interventional techniques in chronic spinal pain.
Part II: guidance and recommendations. Pain Physician 16(2 Suppl):S49-S283

59. Manchikanti L, Boswell MV, Singh V, Derby R, Fellows B, Falco FJ, Datta S, Smith HS, Hirsch JA (2009) Comprehensive review of neurophysiologic basis and diagnostic interventions in managing chronic spinal pain. Pain Phys 12(4):E71-E120 (Review)

60. Manchikanti L, Buenaventura RM, Manchikanti KN, Ruan X, Gupta S, Smith HS, Christo PJ, Ward SP (2012) Effectiveness of therapeutic lumbar transforaminal epidural steroid injections in managing lumbar spinal pain. Pain Phys 15(3):E199-E245 (Review)

61. Manchikanti L, Datta S, Gupta S, Munglani R, Bryce DA, Ward SP, Benyamin RM, Sharma ML, Helm S 2nd, Fellows B, Hirsch JA (2010) A critical review of the American Pain Society clinical practice guidelines for interventional techniques: part 2. therapeutic interventions. Pain Phys 13(4):E215-E264 (Review)

62. Manchikanti L, Singh V, Vilims BD, Hansen HC, Schultz DM, Kloth DS (2002) Medial branch neurotomy in management of chronic spinal pain systematic review of the evidence. Pain Physician 5(4):405-418

63. Mense SS (2004) Funktionelle Neuroanatomie und Schmerzreize: Aufnahme, Weiterleitung und Verarbeitung. Schmerz 18:225-237. https://doi. org/10.1007/s00482-003-0297-8

64. Nguyen C, Boutron I, Baron G, Sanchez K, Palazzo C, Benchimol R, Paris G, James-Belin É, LefèvreColau MM, Beaudreuil J, Laredo JD, Béra-Louville A, Cotten A, Drapé JL, Feydy A, Ravaud P, Rannou F, Poiraudeau S (2017) Intradiscal glucocorticoid injection for patients with chronic low back pain associated with active discopathy: a randomized trial. Ann Intern Med 166(8):547-556. https://doi org/10.7326/M16-1700

65. Niemiec K (2015) Manuelle Medizin in der Behandlung von Rückenschmerzen. Eine kritische Bestandsaufnahme. Man Med 53:424-446

66. Nijs J, Apeldoorn A, Hallegraeff $\mathrm{H}$, Clark J, Smeets R, Malfliet A, Girbes EL, De Kooning M, Ickmans K (2015) Low back pain: guidelines for the clinical classification of predominant neuropathic, nociceptive, or central sensitization pain. Pain Phys 18(3):E333-E346 (Review)

67. Olmarker K, Rydevik B, Kikuchi S (2006) Sciatica and nerve root pain in disc herniation and spinal stenosis: a basic science review and clinical perspective. In:Herkowitz HN, Garfin SR, EismontFJ (Hrsg) Rothman-Simeone-the spine, 5. Aufl. Saunders, , S94-107. ISBN 978-0-323-39397-3

68. Orrock PJ, Myers SP (2013) Osteopathic intervention in chronic non-specific low back pain: a systematic review. BMC Musculoskelet Disord 14:129. https://doi.org/10.1186/1471-2474-14129 (Review)

69. Ozcan S, Muz A, Altun YA, Onal SA (2018) Intradiscal ozone therapy for lumbar disc herniation. Cell Mol Biol (Noisy-le-grand) 64(5):52-55

70. Paige NM, Miake-Lye IM, Booth MS, Beroes JM, Mardian AS, Dougherty P, Branson R, Tang B, Morton SC, ShekellePG (2017) Association of spinal manipulative therapy with clinical benefit and Harm for acute low back pain: systematic review and meta-analysis. JAMA 317(14):1451-1460. https://doi.org/10.1001/jama.2017.3086 (Review)

71. Parr AT, DiwanS, AbdiS (2009) Lumbarinterlaminar epidural injections in managing chronic low back and lower extremity pain: a systematic review. Pain Phys 12(1):163-188 (Review)
72. Parr AT, Manchikanti L, Hameed H, Conn A, Manchikanti KN, Benyamin RM, Diwan S, Singh V, Abdi S (2012) Caudal epidural injections in the management of chronic low back pain: a systematic appraisal of the literature. Pain Phys 15(3):E159-E198 (Review)

73. Perolat $R$, Kastler A, Nicot B, Pellat JM, Tahon F, Attye A, Heck O, Boubagra K, Grand S, Krainik A (2018) Facet joint syndrome: from diagnosis to interventional management. Insights Imaging 9(5):773-789. https://doi.org/10.1007/s13244018-0638-x (Review)

74. Peterson C, Hodler J (2010) Evidence-based radiology (part 1): Is there sufficient research to support the use of therapeutic injections for the spine and sacroiliac joints? Skelet Radiol 39(1):5-9. https://doi.org/10.1007/s00256-0090783-x (Review)

75. Pongratz G (2020) Spezielle Schmerztherapie bei rheumatischen Erkrankungen. Akt Rheumatol 45:430-442. https://doi.org/10.1055/a-12035172

76. Quraishi NA (2012) Transforaminal injection of corticosteroids for lumbar radiculopathy: systematic review and meta-analysis. Eur Spine J 21(2):214-219. https://doi.org/10.1007/s00586011-2008-y (Review)

77. Rho ME, Tang CT (2011) The efficacy of lumbar epidural steroid injections: transforaminal, interlaminar, and caudal approaches. Phys Med Rehabil Clin N Am 22(1):139-148. https://doi.org/10.1016/ j.pmr.2010.10.006

78. Roberts ST, Willick SE, Rho ME, Rittenberg JD (2009) Efficacy of lumbosacral transforaminal epidural steroid injections: a systematic review. PMR 1(7):657-668.https://doi.org/10.1016/j.pmrj. 2009.04.008

79. Rubinstein SM, Terwee CB, Assendelft WJ, de Boer MR, van Tulder MW (2012) Spinal manipulative therapy for acute low-back pain. Cochrane Database Syst Rev. https://doi.org/10. 1002/14651858.CD008880.pub2

80. Saal JS (2002) General principles of diagnostic testing as related to painful lumbar spine disorders: a critical appraisal of current diagnostic techniques. Spine 27(22):2538-2545. https://doi.org/10.1097/ 00007632-200211150-00027 (discussion 2546. Review)

81. Sanzarellol, Merlini L, Rosa MA, Perrone M, Frugiuele J, Borghi R, Faldini C (2016) Central sensitization in chronic low back pain: a narrative review. J Back Musculoskelet Rehabil 29(4):625-633. https://doi. org/10.3233/BMR-160685 (Review)

82. Schmid G, Schmitz A, Borchardt D, Ewen K, von Rothenburg T, Koester O, Jergas M (2006) Effective dose of CT- and fluoroscopy-guided perineural/epidural injections of the lumbar spine: a comparative study. Cardiovasc Intervent Radiol 29(1):84-91. https://doi.org/10.1007/s00270004-0355-3

83. Schütz U (2010) Komplikationen bei der bildgebenden Diagnostik. In: Wirth CJ, Mutschler W, Bischoff HP, Püschmann H, Neu J (Hrsg) Komplikationen in Orthopädie und Traumatologie. Vermeiden, erkennen, behandeln. Thieme, Stuttgart, S72-81. ISBN 978-3-13-148751-3

84. Schütz U (2015) Komplikationen bei der bildgebenden Diagnostik. In: Wirth CJ, Mutschler W, Bischoff HP, Püschmann H, Neu J (Hrsg) Komplikationen kompakt: Orthopädie und Unfallchirurgie. Thieme, Stuttgart, S 30-39. ISBN 978-3-13199091-4

85. Schütz U (2018) Moderne bildgebende Diagnostik der Arthrose im Allgemeinen und der großen 
Gelenke der unteren Extremität. OUP 7:349-365. https://doi.org/10.3238/oup.2018.0349-0365

86. Schütz U (2019) Moderne radiologische Diagnostik in der Wirbelsäulenchirurgie. Orthopade 48(1):5-43. https://doi.org/10.1007/s00132-01803672-8 (Review)

87. Schütz U, Beer M, Wild A, Oehler S, Kraus M (2016) Strahlenschutz bei C-Bogen-gestützten Wirbelsäulenprozeduren in Orthopädie und Unfallchirurgie. OUP 4:224-237. https://doi.org/ 10.3238/oup.2015.0224-237 (Review)

88. Schütz U, Cakir B, Dreinhöfer K, Richter M, Koepp H (2011) Diagnostic value of lumbar facet joint injection: a prospective triple cross-over study. Plos One 6(11):e27991. https://doi.org/10.1371/ journal.pone.0027991

89. Schütz U, Kraus M (2019) Strahlenschutz und C-Bogen-Bedienung. In: Jerosch J (Hrsg) Minimalinvasive Wirbelsäulenintervention, 3. Aufl. Springer, Berlin Heidelberg, S 55-71. ISBN 978-3662-58093-6 (Part of Springer Nature)

90. Schütz U, Schott C (2017) Infiltrationstherapie und Reflextherapie. In: Psczolla M, Kladny B, Flechtenmacher J, Hoffmann R, Dreinhöfer $K$ (Hrsg) Weißbuch: Konservative Orthopädie und Unfallchirurgie. De Gruyter, Berlin, Boston, S 168-172. ISBN 978-3-11-053433-7

91. Schwarzer AC, Aprill CN, Derby R, Fortin J, Kine G, Bogduk N (1994) Clinical features of patients with pain stemming from the lumbar zygapophysial joints. Is the lumbar facet syndrome a clinical entity? Spine 19(10):1132-1137. https://doi.org/ 10.1097/00007632-199405001-00006

92. Seffinger MA, Najm WI, Mishra Sl, Adams A, Dickerson VM, Murphy LS, Reinsch S (2004) Reliability of spinal palpation for diagnosis of back and neck pain: a systematic review of the literature. Spine 29(19):E413-E425. https://doi.org/10.1097/ 01.brs.0000141178.98157.8e (Review)

93. Simopoulos TT, Manchikanti L, Singh V, Gupta S, Hameed H, Diwan S, Cohen SP (2012) A systematic evaluation of prevalence and diagnostic accuracy of sacroiliac joint interventions. Pain Phys 15(3):E305-E344 (Review)

94. Solomonow M, Zhou BH, Harris M, Lu Y, Baratta RV (1998) The ligamento-muscular stabilizing system of the spine. Spine 23(23):2552-2562. https://doi. org/10.1097/00007632-199812010-00010

95. Soto Quijano DA, Otero Loperena E (2018) Sacroiliac joint interventions. Phys Med Rehabil Clin N Am 29(1):171-183.https://doi.org/10.1016/ j.pmr.2017.09.004 (Review)

96. Staal JB, de Bie R, de Vet HC, Hildebrandt J, Nelemans P (2008) Injection therapy for subacute and chronic low-back pain. Cochrane Database Syst Rev 2008(3):CD1824.https://doi.org/10.1002/ 14651858.CD001824.pub3

97. Tay M,Sian SCSH,EowCZ,HoKLK, Ong JH, Sirisena D (2021) Ultrasound-guided lumbar spine injection for axial and radicular pain: a single institution early experience. Asian Spine J 15(2):216-223. https:// doi.org/10.31616/asj.2019.0399

98. Theodoridis T (2019) Landmarkengestützte Infiltrationen und Injektionstechniken an der Hals-, Brust- und Lendenwirbelsäule. In: Jerosch J (Hrsg) Minimalinvasive Wirbelsäulenintervention. Springer, Berlin, S35-53. ISBN 978-3-662-58093-6

99. Theodoridis T, Krämer J (2017) Injektionstherapie an der Lendenwirbelsäule. In: Theodoridis T, Krämer J (Hrsg) Injektionstherapie an der Wirbelsäule. Manual und Atlas, 3. Aufl. Thieme, Stuttgart, S 119-180. ISBN 978-3-13-141043-6

100. Theodoridis T, Randel U, Georgallas C (2019) Landmarkengestützte Injektionstechniken an der

Therapeutic injections and manual medicine in low-back pain. Bimodal synergies between evidence and empiricism

Background: Oriented towards the therapy planning and management of rheumatic patients, and based on the differentiated therapeutic principles of manual medicine (MM) with knowledge on evidence of therapeutic local infiltration techniques (TLI), the author pleads for the establishment of a structured, mechanism-based therapy concept in the sense of "treat to target" (T2T) for patients with (chronic) degenerative low-back pain (LBP) in outpatient pain therapy care.

Diagnostics: This requires a consistent (primary) diagnosis with pain analysis under the premise that LBP is always specific if it is structurally and functionally conditioned. A broad bio-psycho-social anamnesis and structure-based clinical diagnosis (imaging) with functional differentiation according to MM principles and, if necessary, interventional blocks, should result in the expression of a three-level diagnosis as a prerequisite for a mechanism-based, hierarchic step therapy in LBP. In this article, this is presented in a pragmatic, case-oriented manner, with the implementation of techniques and evidence of TLI and MM.

Keywords

Chronic pain · Injection - Lumbago · Sacroiliac joint · Spine

Wirbelsäule. OUP 8:528-539. https://doi.org/10. 3238/oup.2019.0528-0539

101. van Tulder $M$, Becker A, Bekkering T, Breen $A$, del Real MT, Hutchinson A, Koes B, Laerum E, Malmivaara A, COST B13 Working Group on Guidelines for the Management of Acute Low Back Pain in Primary Care (2006) Chapter 3. European guidelines for the management of acute nonspecific low back pain in primary care. Eur Spine J 15(Suppl 2):169-191. https://doi.org/10. 1007/s00586-006-1071-2

102. Verhagen AP, Downie A, Popal $N$, Maher C, Koes BW (2016) Red flags presented in current low back pain guidelines: a review. Eur Spine J 25(9):2788-2802. https://doi.org/10.1007/s00586-016-4684-0 (Review)

103. Wancura-Kampik I (2014) Referred pain. In: Wancura-Kampik I (Hrsg) Segmental anatomy: the key to mastering acupuncture, neural therapy and manual therapy. Urban \& Fischer Elsevier, München, S249-278. ISBN 978-3-437-57972-1

104. Waseem Z, Boulias C, Gordon A, Ismail F, Sheean G, Furlan AD (2011) Botulinum toxin injections for low-back pain and sciatica. Cochrane Database Syst Rev 19(1):CD8257. https://doi.org/10.1002/ 14651858.CD008257.pub2 (Review)

105. Watts RW, Silagy CA (1995) A meta-analysis on the efficacy of epidural corticosteroids in the treatment of sciatica. Anaesth Intensive Care 23(5):564-569. https://doi.org/10.1177/0310057X9502300506

106. Waurick K, Zarbock A, Gogarten W, Kessler P, Volk T, Steinfeldt T, Wiesmann T, Riess H (2021) Rückenmarksnahe Regionalanästhesien und Thrombembolieprophylaxe/antithrombotische Medikation. 3. überarbeitete Empfehlung der Deutschen Gesellschaft für Anästhesiologie und Intensivmedizin (DGAI). AWMF-Register-Nr.: 001 005, Klassifikation S1. http://www.leitlinien.de/ themen/kreuzschmerz/2-auflage.Zugegriffen: 6 . Jan. 2022

107. Weiterbildungsordnung 2018 der Bundesärztekammer in der Fassung vom 26.06.2021. http://www.bundesaerztekammer.de/aerzte/ aus-weiter-fortbildung/weiterbildung/musterweiterbildungsordnung.Zugegriffen: 1.Jan.2022
108. Won HS, Yang M, Kim YD (2020) Facet joint injections for management of low back pain: a clinically focused review. Anesth Pain Med 15(1):8-18. https://doi.org/10.17085/apm.2020. 15.1.8 (Review)

109. Mendoza JE (2011) Dermatome. In: Kreutzer JS, DeLuca J, Caplan B (Hrsg) Encyclopedia of clinical neuropsychology. Springer, New York https://doi. org/10.1007/978-0-387-79948-3_725 
Hier steht eine Anzeige.

黑 Springer 